\title{
Archéologie et hydrogéologie
}

De nouvelles données concernant la gestion de l'eau dans la cité de Solonte (nord-ouest Sicile, Italie)

Giovanni Polizzi, Vincent Ollivier, Iván Fumadó Ortega et Sophie Bouffier

\section{(2) OpenEdition}

\section{Journals}

Édition électronique

URL : https://journals.openedition.org/cefr/1705

DOI : $10.4000 /$ cefr. 1705

ISSN : 2282-5703

Éditeur

École française de Rome

Référence électronique

Giovanni Polizzi, Vincent Ollivier, Iván Fumadó Ortega et Sophie Bouffier, « Archéologie et

hydrogéologie », Chronique des activités archéologiques de l'École française de Rome [En ligne], Sicile, mis en ligne le 12 avril 2017, consulté le 13 avril 2022. URL : http://journals.openedition.org/cefr/1705 :

DOI : https://doi.org/10.4000/cefr.1705

Ce document a été généré automatiquement le 13 avril 2022.

(c) École française de Rome 


\section{Archéologie et hydrogéologie}

De nouvelles données concernant la gestion de l'eau dans la cité de Solonte (nord-ouest Sicile, Italie)

Giovanni Polizzi, Vincent Ollivier, Iván Fumadó Ortega et Sophie Bouffier

\section{NOTE DE L'AUTEUR}

Ce travail a bénéficié d'une aide du gouvernement français, gérée par l'Agence Nationale de la Recherche au titre du projet Investissements d'Avenir A*MIDEX portant

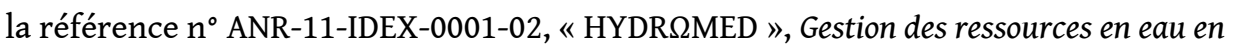
Méditerranée pendant le $1^{\text {er }}$ millénaire avant notre ère, coordonné par Sophie Bouffier (CCJ, UMR 7299). Nous remercions les responsables du Parc archéologique de Solonte (L. Fricano, ex directrice du parc archéologique, F. Spatafora, directrice actuelle du Polo regionale di Palermo per i parchi e i musei archeologici, dont le parc archéologique de Solunto fait partie, et Laura Di Leonardo, fonctionnaire du parc archéologique de Solunto) de même que l'ensemble de ses gardiens pour leur soutien constant. Nous souhaitons également remercier Vincent Dumas (CCJ, UMR 7299) pour la réalisation des orthophotographies des thermes, ainsi que Frerich Schoen et Stefano Cespa de la KarlEberhard Universität Tübingen pour leur coopération.

1 Le programme sur la gestion de l'eau dans la ville antique de Solonte (Santa Flavia, PA) a été lancé dans le cadre du réseau HYDR $\Omega$ MED (http://hydromed.hypotheses.org/), dirigé par Sophie Bouffier (Centre Camille Jullian, Aix-Marseille Université) et financé par la fondation $\mathrm{A}^{*}$ Midex. Interdisciplinaire, ce projet a pour finalité une meilleure compréhension des différentes modalités d'adaptation des populations antiques à l'environnement méditerranéen en mettant l'accent sur les ressources en eau.

Connu dès le XVIe siècle, le site de Solonte, localisé sur le promontoire du Monte Catalfano, a été fouillé épisodiquement à partir de 1825 et de manière intensive entre les années 1950 et 1960 sous la direction de V. Tusa ${ }^{1}$. On y avait mis au jour une zone publique et des quartiers d'habitation, organisés selon un plan régulier, que les habitants ont approvisionnés en eau par le biais d'un système complexe de 
canalisations et de réservoirs permettant d'exploiter au mieux les conditions environnementales du secteur. Les récentes recherches se sont concentrées sur le mobilier mais n'ont pas concerné la question de la gestion des eaux, abordée rapidement dans un guide archéologique, Solunto, publié en 1994. Le programme interdisciplinaire lancé par des chercheurs d'Aix-Marseille Université (Centre Camille Jullian, LAMPEA) à l'été 2016 a pour objectif de mettre en lumière les différentes modalités d'adaptation de la population solontine à ses ressources naturelles, en portant plus particulièrement l'attention sur la gestion de l'eau ${ }^{2}$. C'est la première fois depuis les premières campagnes de fouilles de 1825 qu'une étude associant hydrogéologie, géomorphologie et archéologie est menée sur ce site. L'état des lieux avait mis en évidence un approvisionnement fondé sur un recours massif, voire exclusif, aux citernes maçonnées, 76 exemplaires dans l'espace urbain, d'où l'idée couramment répandue que le site manquait d'eau et était alimenté uniquement par les précipitations.

3 La campagne de l'été 2016 a donc présenté deux volets : une prospection et une étude géomorphologiques pour mettre en évidence les caractéristiques hydrogéologiques du Monte Catalfano et définir ainsi le rapport entre l'hydrogéologie et l'architecture lors des choix de gestion hydraulique; l'étude de deux cas particuliers: la citerne en maçonnerie et le système d'alimentation des thermes.

\section{Hydrogéologie, géoarchéologie et gestion de l'eau dans la cité de Solonte : des liens évidents ?}

Les premières analyses géoarchéologiques et hydrogéologiques ont permis de révéler un important potentiel de recherches interdisciplinaires concernant l'alimentation et la gestion de l'eau dans la cité phénico-punique. Nos premières données soulignent l'importance de l'hydrogéologie locale dans l'origine des eaux alimentant la cité. La situation géologique singulière de la Sicile, dans la zone de contact entre la plaque africaine et la plaque eurasienne, de même que sa localisation dans le domaine climatique méditerranéen sont des composantes (morphostructurales et climatiques) conditionnant l'ensemble des paramètres hydrogéologiques et hydrologiques intégrés dans notre étude.

Le massif de Monte Catalfano est un modeste relief d'orientation nord-sud avoisinant les $235 \mathrm{~m}$ a.s.l. marqué par de fortes pentes (déclivité moyenne de $32 \%$ ) et falaises (pente moyenne de $75 \%$ ) regardant la mer Tyrrhénienne. Du point de vue lithologique, il correspond principalement dans notre secteur à un ensemble calcaire bréchique et dolomitique (au pendage de $40^{\circ}$ vers le sud-ouest) dont la série du Lias (Jurassique Inférieur) avoisine les 300 mètres de puissance ${ }^{3}$. Cette unité, appartenant structuralement aux Monts de Palerme, est intensément fracturée par une tectonique cassante relativement active (fig. 1). La moyenne annuelle des précipitations sur le massif est de $650 \mathrm{~mm}^{4}$. Celles-ci se produisent essentiellement en hiver tandis que l'intégralité de la période estivale est particulièrement sèche (4 $\mathrm{mm}$ en moyenne pour le mois de juillet : climate-data.org). 
Fig. 1 - Carte géologique du Monte Catalfano.

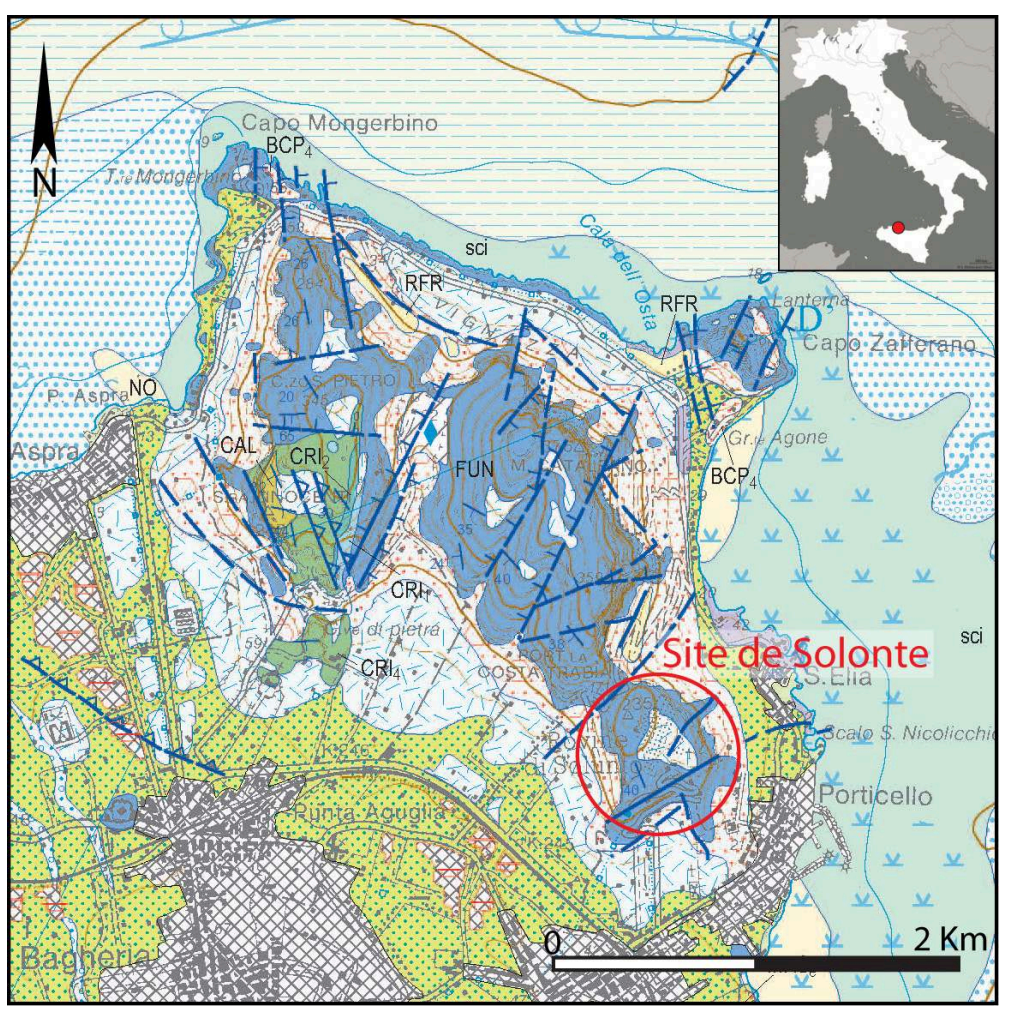

Modifié d'après Catalano et alii 2013.

Compte tenu de la nature dolomitique et bréchique des affleurements, offrant une porosité supérieure aux seuls calcaires, et en association avec une importante fracturation, le massif de Monte Catalfano dispose d'une perméabilité spécifique significative et d'une grande capacité de stockage et de recharge en eaux météoriques ${ }^{5}$.

Les données de terrain ont permis d'identifier une série d'accidents tectoniques principaux généralement orientés NE-SO. Mais plus important, plusieurs fracturations en faisceaux secondaires décrochant d'orientation $\mathrm{N} 250^{\circ}$ à $\mathrm{N} 325^{\circ}$ présentent des laminations calcitiques de type hydrothermal (ascendance d'eaux profondes) venant combler les plans de faille (fig. 2). Ces minéralisations indiquent un contrôle de la fracturation sur la géométrie de circulations des fluides et soulignent le potentiel carbo-gazeux local lié à un hydrothermalisme associé, dans la baie toute proche, à des phénomènes paravolcaniques (volcans de boue ${ }^{6}$ ). De plus, à proximité de Pizzo Cannita et de Villabate, à moins de 5 kilomètres du Monte Catalfano, une activité hydrothermale est également signalée dans un texte datant du XVI ${ }^{e}$ siècle ${ }^{7}$. 
Fig. 2 - Détail sur les remontées hydrothermales à développement de laminations calcitiques le long des failles affectant le massif calcaire et dolomitique du Monte Catalfano.

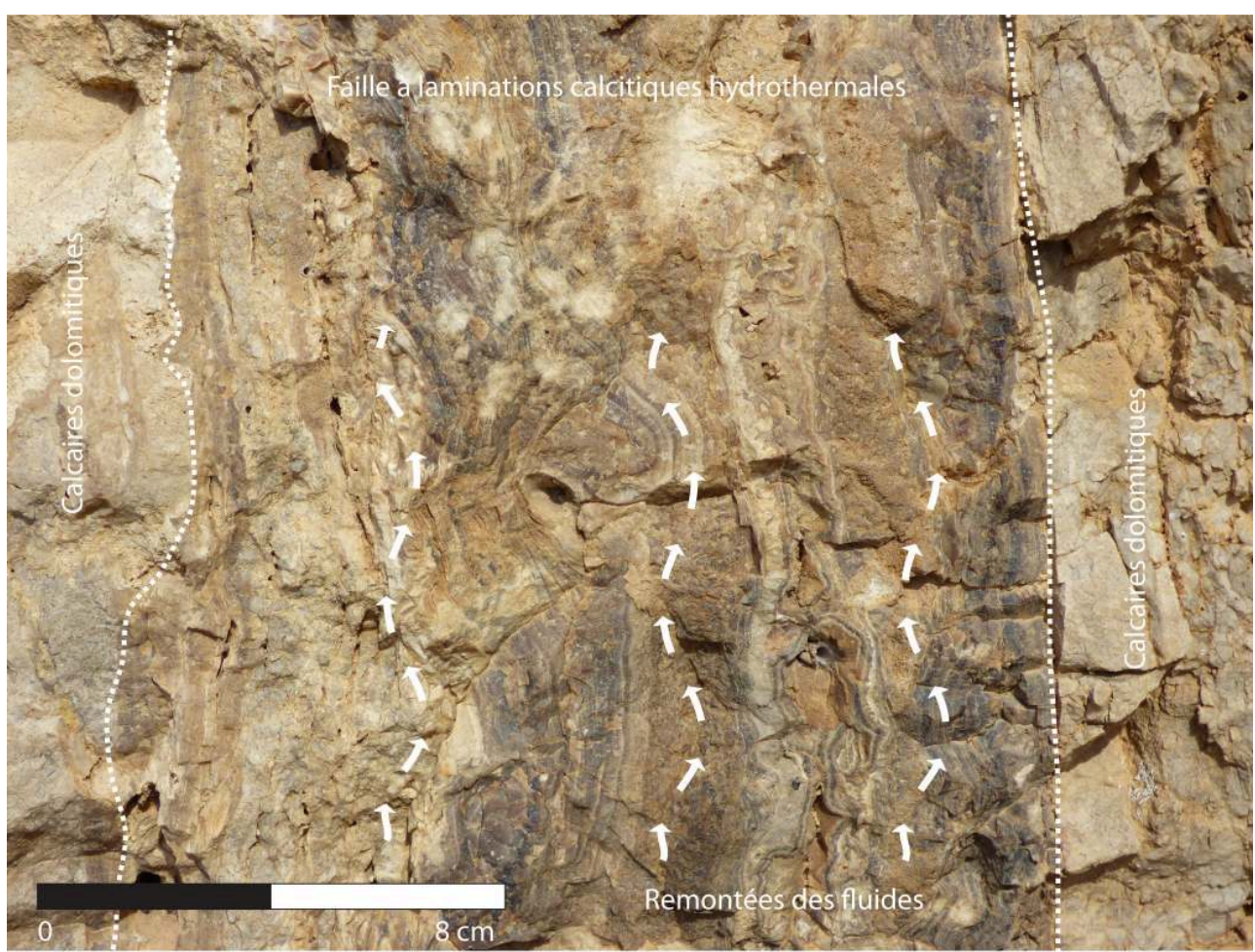

Photo V. Ollivier.

7 À Solonte, ces circulations ascendantes de fluides semblent avoir fonctionné à l'échelle géologique mais certaines d'entre elles ont été actives lors de l'occupation du Monte Catalfano. En effet, plusieurs filons hydrothermaux montrent des laminations et concrétionnements de parois et structures archéologiques. Des suintements actuellement actifs et à faibles encroûtements sont également visibles en plusieurs endroits du site (fig. 3). 
Fig. 3 - Émergences actuelles le long des failles hydrothermales et développement de concrétionnements algo-bryophytiques sur le site de Solonte.

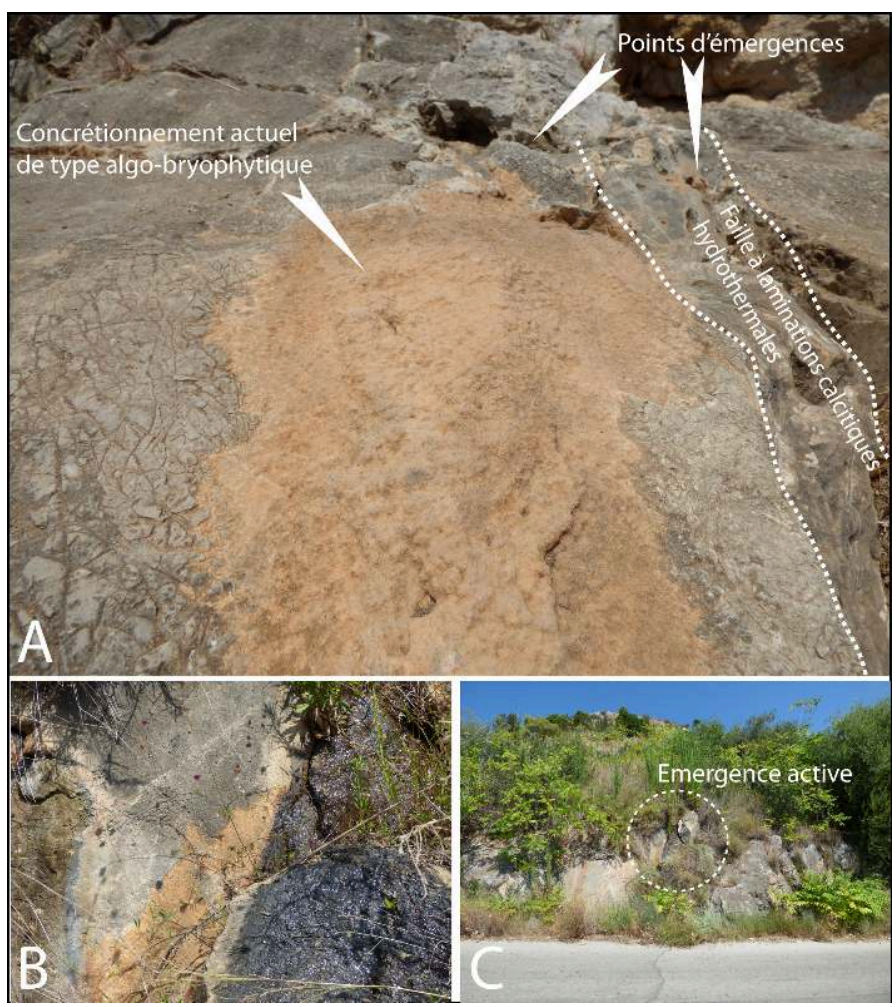

Thermes et voie d'accès.

Photos V. Ollivier.

$\mathrm{Au}$ niveau des thermes, des concrétionnements laminés moulent les conduites principales du bâti (fig. 4) témoignant du caractère juvénile des eaux tout en écartant une origine liée aux seules eaux de pluies. Dans ce cas, il apparaît qu'une importante partie des eaux alimentant la cité et notamment les thermes, devait provenir de ces résurgences de type hydrothermal ce qui modifie substantiellement les hypothèses antérieures. 
Fig. 4 - Détail sur les concrétionnements laminés des thermes de Solonte.

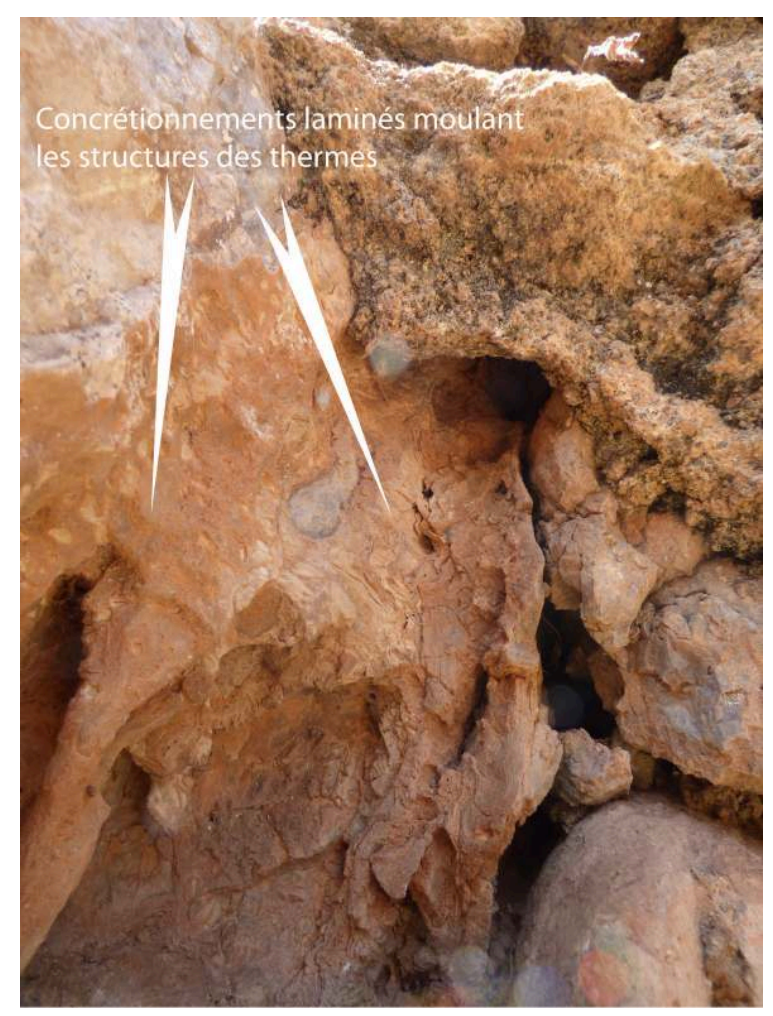

Photo V. Ollivier.

8 Un lien entre les différentes fracturations secondaires du massif et la répartition des dispositifs de stockage des eaux (bassins, citernes) n'est également pas à écarter. Plusieurs observations permettent de constater que dans de nombreux cas, ces derniers sont associés aux failles à filons hydrothermaux dont les eaux viendraient alimenter, conjointement aux eaux de pluies, ces différentes structures. Un travail de cartographie précise des relations entre failles à remontées hydrothermales et citernes/bassins est en cours de finalisation. Une analyse microscopique des séries de lamines afin de déterminer l'origine et la rythmicité des concrétionnements est réalisée à la fois sur les gîtes initiaux mais également sur les précipitations carbonatées contemporaines du site. Enfin, des prélèvements pour analyses géochimiques (isotopes du plomb, du strontium, différents éléments traces et datations U/Th) ont été effectués sur les lamines calcitiques/dolomitiques. Ces derniers sont en cours de traitement au CEA/ LSCE (UMR 8212, Paris, France) afin de mieux caractériser l'origine des eaux.

\section{L'alimentation en eau des citernes : nouvelles données}

La collecte de l'eau dans l'environnement du Mont Catalfano qui apparaissait comme un site propice à l'implantation d'un établissement, s'est faite par un réseau de citernes bien distribuées sur tout le site. Chaque édifice dispose au moins d'une ou deux citernes (fig. 5), lesquelles peuvent avoir des formes différentes -bi-absidale, ovale, rectangulaire, atypique - et une capacité généralement comprise entre 10 et $30 \mathrm{~m}^{3}$. Ces citernes étaient réalisées en maçonnerie sur le côté aval et présentaient des parois 
amont et un fond creusés dans la roche ou épousant les contours naturels karstifiés. Un mortier hydraulique était appliqué à l'intérieur du réservoir.

Fig. 5 - Photo satellitaire de Solonte avec la localisation des citernes.

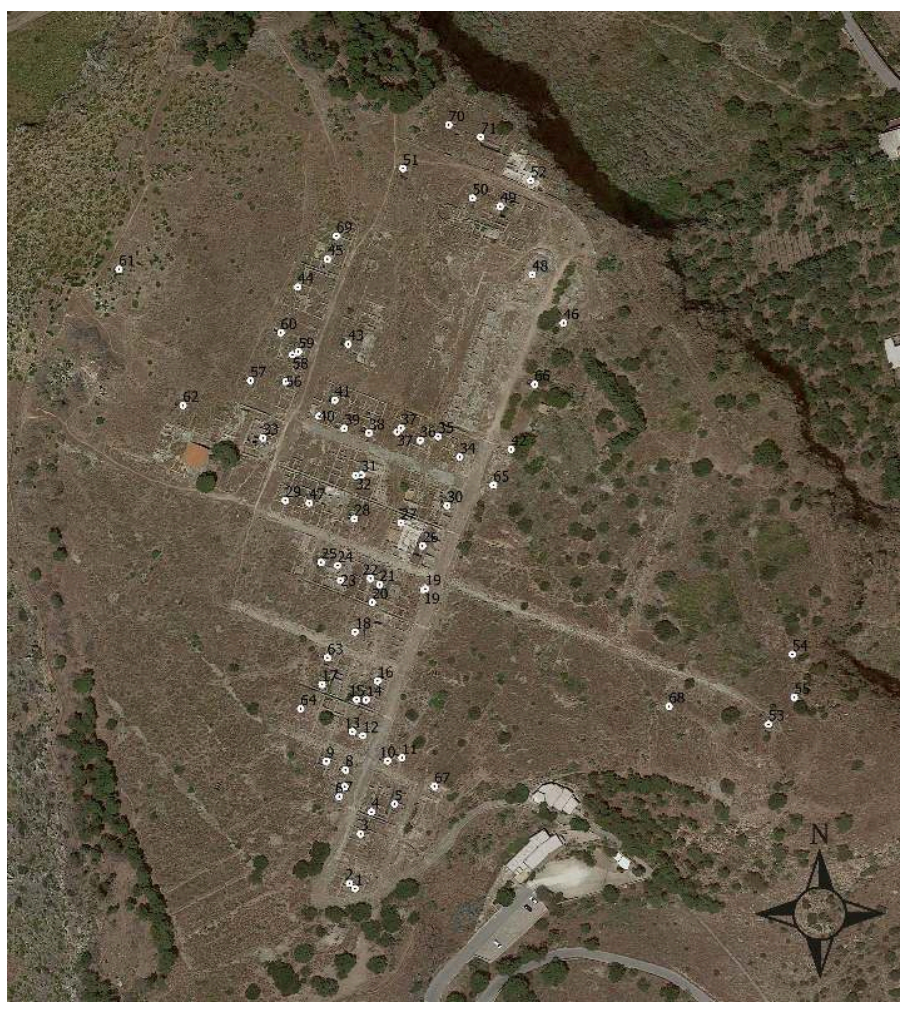

Modifié d'après Schoen 2015.

10 La confrontation des données géomorphologiques et archéologiques nous permet de mettre en relation la répartition des citernes et la présence de petites résurgences hydrothermales localisées au niveau des plans de failles, celles-ci pouvant fournir une alimentation additionnelle aux eaux pluviales récoltées par ruissellement sur le toit des habitations. En effet,

1) Il existe une forte corrélation spatiale entre la localisation des citernes et des fracturations à remontées d'eaux juvéniles.

2) La forme des réservoirs est irrégulière et orientée longitudinalement selon les plans de failles favorisant les résurgences de type hydrothermal.

3) Leur fond est très étroit et irrégulier, reprenant parfois les morphologies karstifiées en surface des failles (dissolution de la roche et ouverture extensive du plan de fracturation $\left.{ }^{8}\right)$.

11 Ces caractéristiques sont communes à la plupart des structures, ce qui nous permet d'envisager une probable relation entre les phénomènes de résurgences et la collecte des eaux.

12 Nous avons pu observer que certaines citernes, également localisées sur l'axe des fracturations du massif, ont un niveau d'eau relativement constant au cours de l'année malgré la modeste pluviométrie régionale et l'existence de longues périodes sèches estivales. Il s'agit des citernes $n^{\circ} 41,57$ et 70 (fig. 5), localisées dans différents secteurs $\mathrm{du}$ site. Ceci indique, tout comme la présence de suintements visibles sur des affleurements et failles le long du chemin d'accès au site (en l'absence mensuelle de 
précipitation ${ }^{9}$ ), que les différents accidents tectoniques référencés favorisent encore aujourd'hui les remontées de type hydrothermal.

\section{L'alimentation en eau des bains}

Les petits thermes, datés du $\mathrm{I}^{\mathrm{er}}$ siècle av. J.-C. ${ }^{10}$, se trouvent topographiquement en aval de la zone d'habitat, au début de la Via dell'Agorà, au niveau de son croisement avec la Via delle Terme (fig. 6). Les structures occupent une terrasse presque triangulaire dont le côté oriental n'est pas conservé. Du point de vue planimétrique le bâtiment présente un schéma axial, avec une distribution des pièces en enfilade dans le sens nord-sud.

Fig. 6 - Plan des thermes.

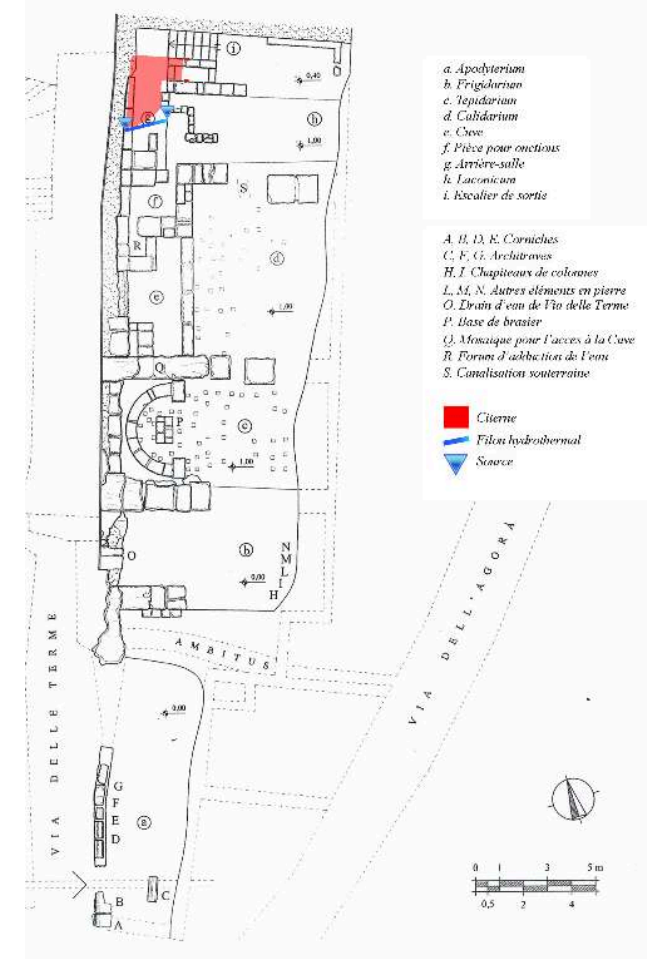

D’après Sposito 2014

On a toujours pensé que l'alimentation en eau des thermes dépendait du bâtiment en amont, où se trouve la grande citerne $n^{\circ} 5$ (fig. 5), et des ambitus qui entourent l'édifice. En réalité il n'a pas été possible de retrouver des éléments d'adduction tant au niveau des ambitus, que de la citerne amont. De plus, il est difficile d'envisager que l'eau des ambitus ait pu être utilisée à l'intérieur des thermes. Il s'agit sans doute d'une eau insalubre. Il faut donc considérer plus probable l'existence de réservoirs d'eau situés directement dans les thermes. En effet, les thermes disposent d'une citerne juste en amont de la pièce septentrionale ${ }^{11}$ (fig. 7). 
Fig. 7 - La Citerne des thermes (n. 67) vue du nord-ouest, in primo piano la structure tardive.

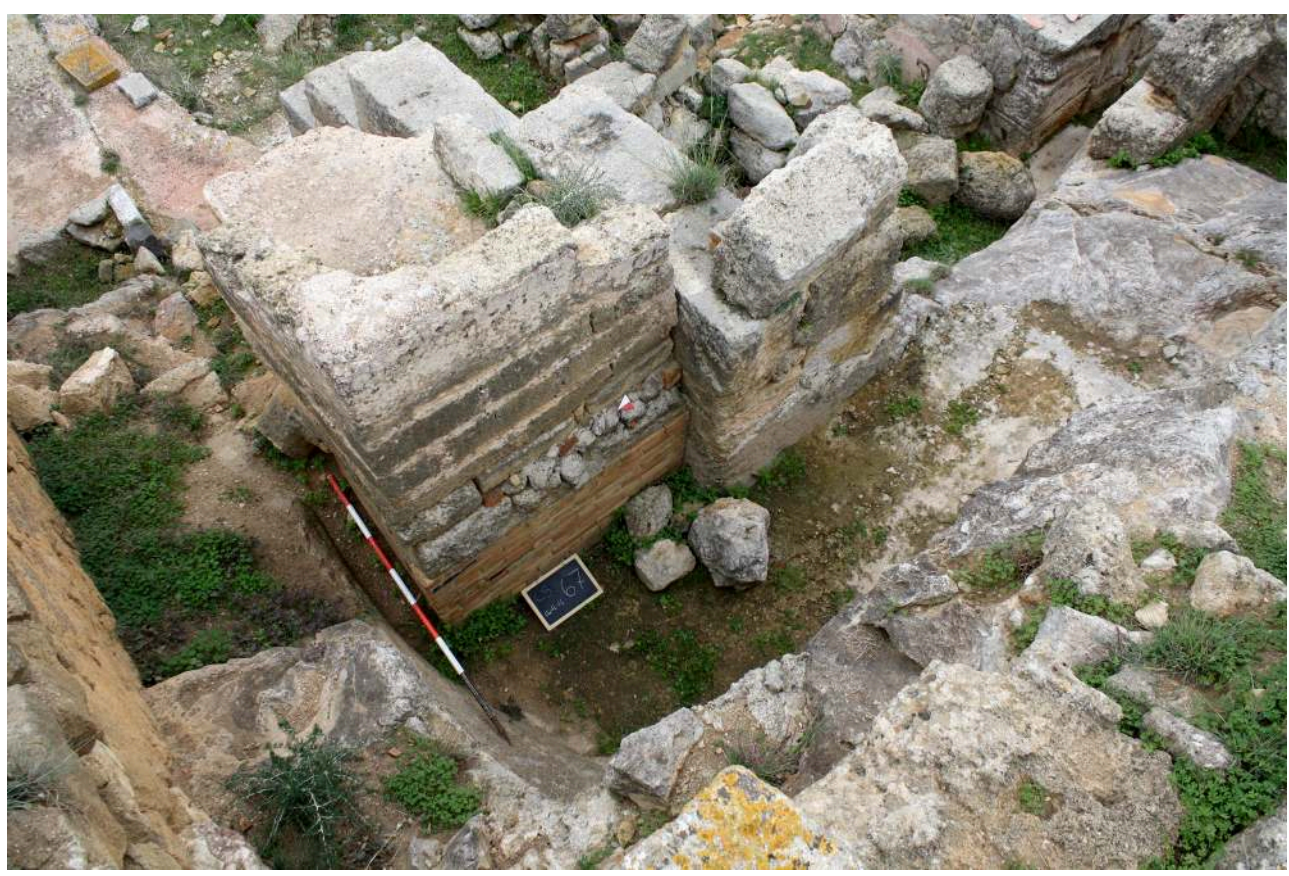

Photo G. Polizzi.

Il s'agit d'un réservoir en forme de L, creusé dans la roche en amont et dont la partie orientale est réalisée dans une maçonnerie utilisant des blocs réguliers de biocalcarénites. La partie orientale de la citerne n'est pas conservée, tout comme la limite orientale du bras est-ouest, oblitérée par une structure tardive. La couverture était réalisée en dalles de pierre, soutenues en partie par un arc dans le secteur nordsud, dont on peut apprécier un bloc au-dessus du mur oriental. La partie creusée dans la roche présente une feuillure facilitant la mise en place d'autres blocs. L'alimentation de la citerne était probablement assurée, conjointement à la collecte de l'eau de pluie, par une source émergeant d'un petit filon hydrothermal traversant le secteur sud des fouilles (fig. 8-9). Celui-ci devait également alimenter les citernes $\mathrm{n}^{\circ} 4$ et $\mathrm{n}^{\circ} 5$. 
Fig. 8 - Orthophotographie de la paroi ouest de la pièce « $h$ » des thermes.

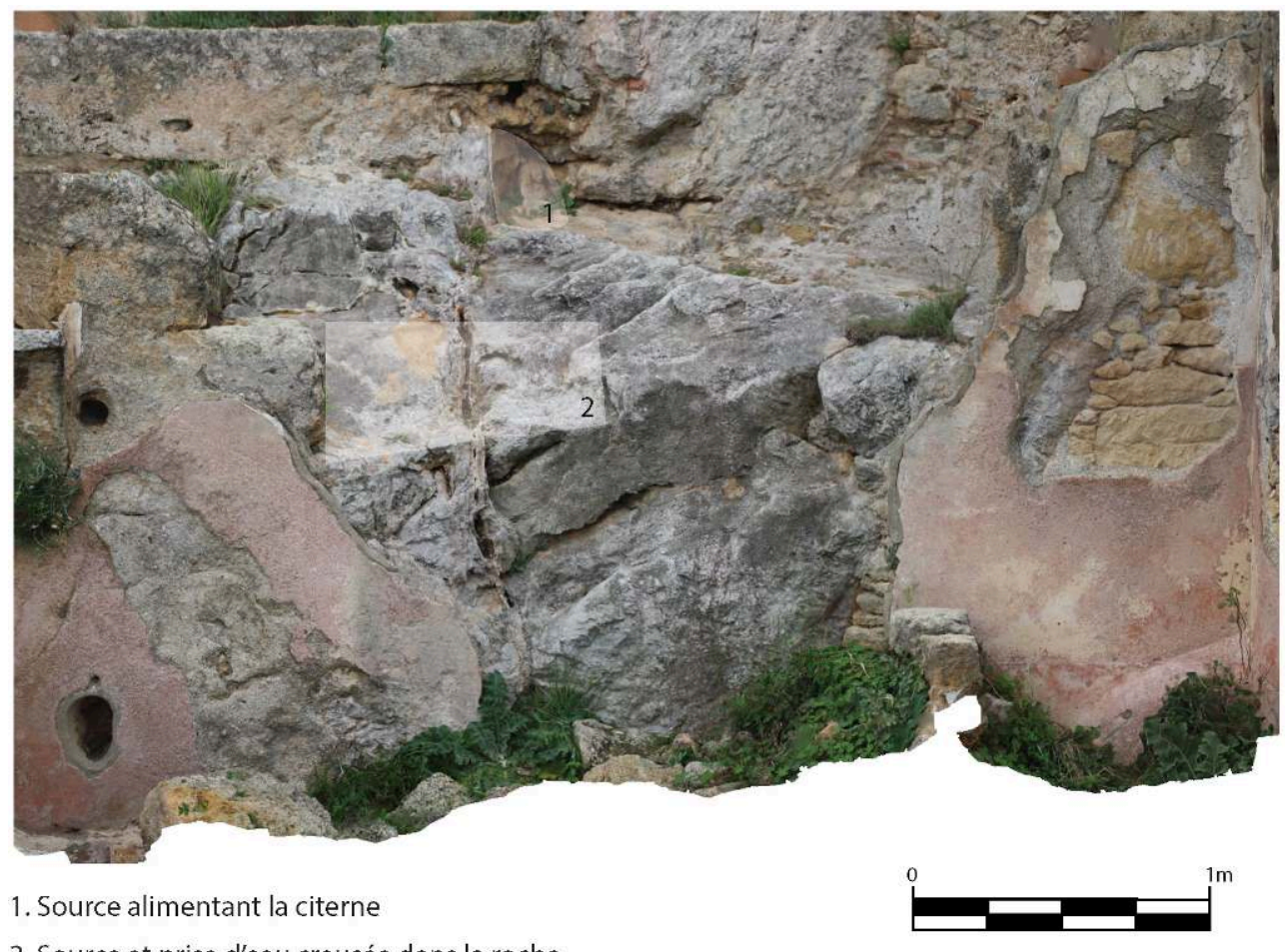

2. Source et prise d'eau creusée dans la roche

Photo G. Polizzi, élaboration V. Dumas.

Fig. 9 - Parois ouest de la pièce « $h$ » des thermes.

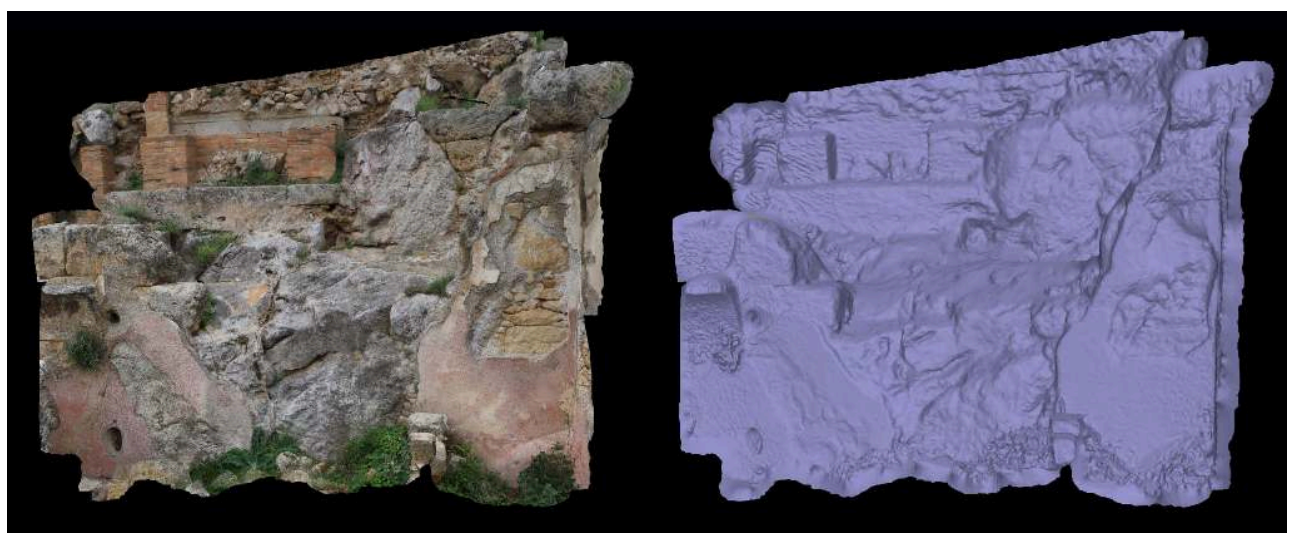

Photo G. Polizzi, élaboration V. Dumas.

Une autre caractéristique de la pièce nord de la structure thermale, qui aujourd'hui est sans parallèles, est la vraisemblable prise d'eau de la paroi ouest (fig. 6h, 8, 9). Celle-ci présente un creusement de la roche le long d'une des principales failles à remontées hydrothermales destiné à capter et canaliser une exsurgence au débit assurément notable, compte tenu de l'épaisseur et de la pureté en calcite des encroûtements laminés observés (fig. 10). 
Fig. 10 - Analyses au microscope digital des laminations et cristallisations au niveau des failles à remontées hydrothermales et de l'émergence des thermes.

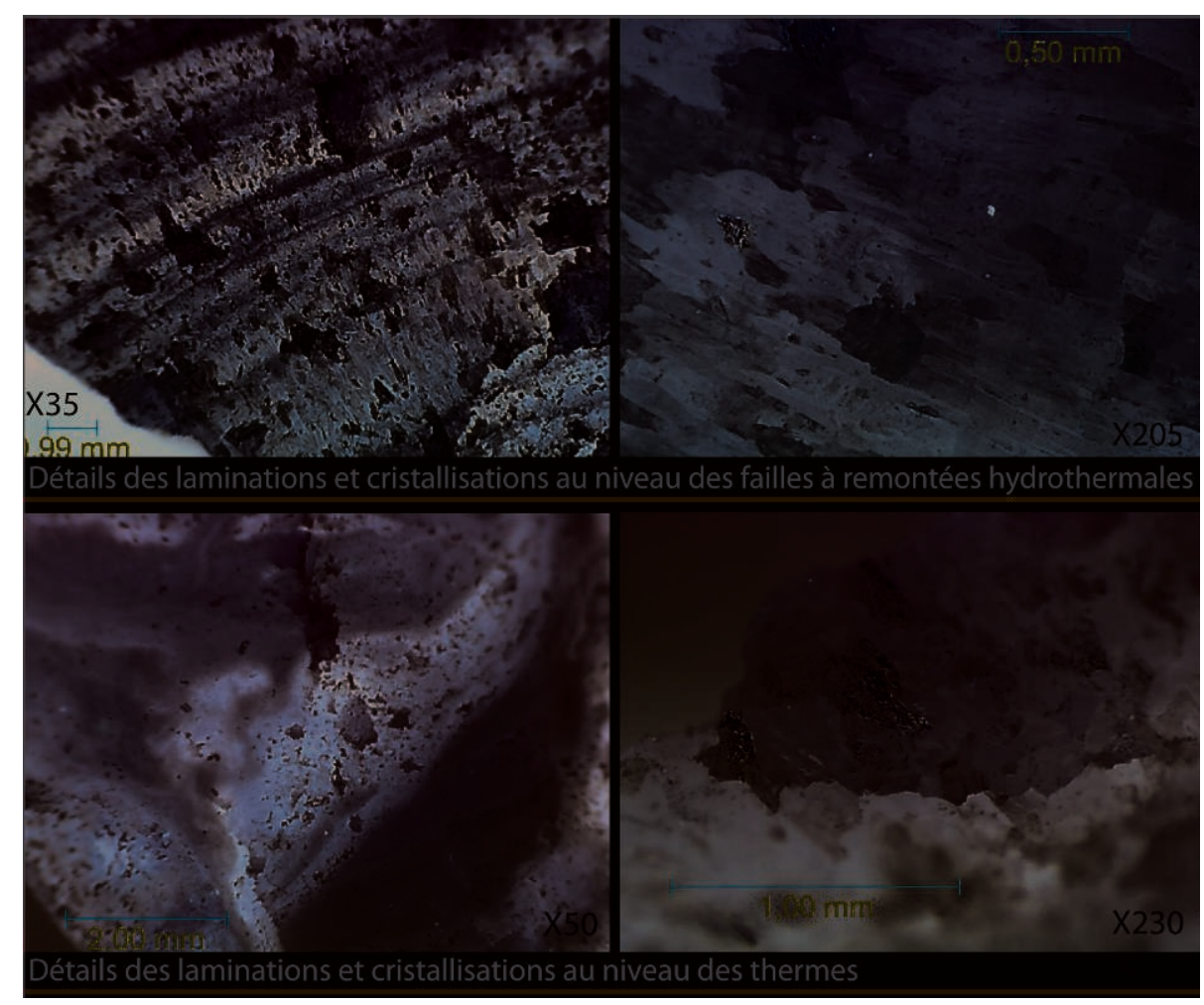

Photos V. Ollivier.

L'aménagement de la pièce, dotée d'un pavement et de parois en béton de tuileau ${ }^{12}$, comprend donc vraisemblablement des émergences au niveau du mur occidental. Cette configuration fait penser à une fonction probablement liée à l'aspersion des baigneurs ou à d'autres pratiques hygiéniques effectuées à la fin du trajet du bain. Il est possible qu'en raison de l'existence de cette exsurgence les thermes aient été bâtis à cet endroit précis. Cette localisation à l'entrée de la ville pouvait être avantageuse en les rendant immédiatement accessibles aux étrangers.

\section{La citerne maçonnée}

17 Afin de mieux comprendre les modalités de stockage de l'eau à Solonte et en complément des analyses hydrogéologiques du site, une approche préliminaire croisée entre archéologie et caractéristiques sédimentologiques des archéomatériaux a été menée sur la citerne en maçonnerie. On lui a d'abord attribué une fonction de type fontaine publique ${ }^{13}$. La karstification d'un plan de fissuration, dans lequel la citerne s'insère, a pu motiver la sélection de cet emplacement. Des indices de remontées hydrothermales et un degré d'humidité constant au cours de l'année y ont été observés et il est possible qu'un petit griffon ait pu en alimenter le fond. De plus amples recherches sur cette question sont bien évidemment nécessaires.

18 Connue en bibliographie comme « cisterna pubblica ${ }^{14}$ » ou « cisterna in muratura ${ }^{15}$ », il s'agit d'un grand réservoir quadripartite situé à l'extrémité occidentale de la partie fouillée de la ville (îlot XII), sur le point culminant du Monte Catalfano. Elle est 
accessible par la plateia nord-sud, Via degli Artigiani et par la plateia est-ouest, Via Salinas (fig. 11).

Fig. 11 - Plan de situation de l'îlot XII de Solonte.

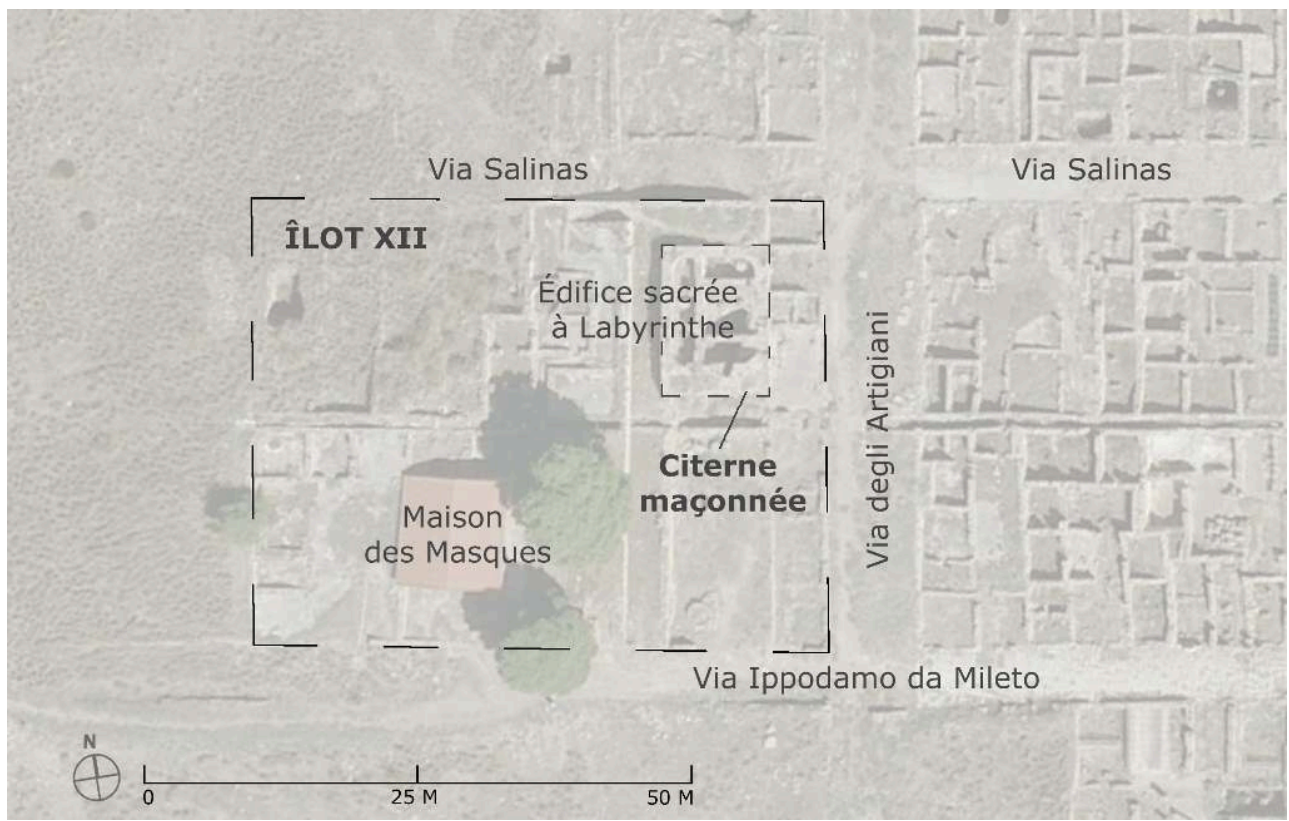

I. Fumadò Ortega.

Fig. 12 - Décoration des parois des thermes (a), de l'agora (b) et de la « Casa del Vano circolare » (c).
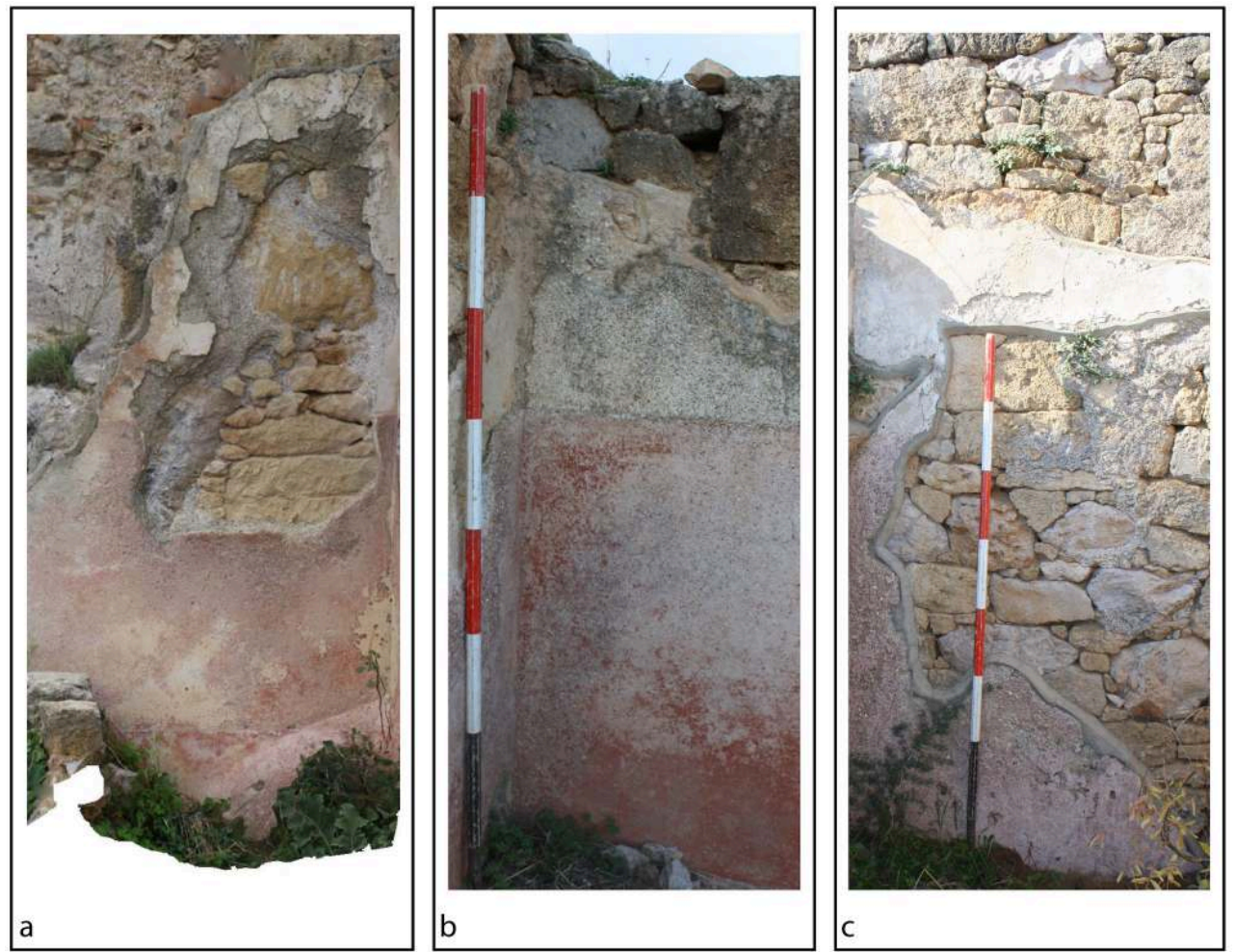

Photo G. Polizzi. 
19 $\mathrm{XX}^{\mathrm{e}}$ siècles. Une première saison de fouilles a été effectuée sous la direction de Francesco Saverio Cavallari entre $1860,1870^{16}$ et $1875^{17}$. Lors de la première campagne de fouilles, entre 1868 et 1869, Giuseppe Patricolo a mis au jour les pièces de la célèbre Maison des Masques ${ }^{18}$, à proximité de la citerne en maçonnerie. La grande citerne avec ses quatre réservoirs, le cryptoportique au nord-ouest, et les escaliers conduisant à l'édifice occidental ont été fouillés dans ce contexte. Après un siècle d'interruption, entre les années 1960 et 1970 la fouille fut reprise par Vincenzo Tusa. Le secteur au sudest de la citerne a pu être défini comme une vaste zone libre pour laquelle on a supposé une destination commerciale ${ }^{19}$, de même que pour les pièces situées à l'est de la citerne. En dehors d'un relevé axonométrique ${ }^{20}$ aucun document concernant ces fouilles n'a pu être retrouvé.

D'un point de vue architectural, les observations faites à partir du relevé direct des murs de la citerne maçonnée (fig. 13) intéressent la structure et les phases de construction de cette citerne ${ }^{21}$.

Fig. 13 - Plan de la citerne maçonnée et des pièces qui l'entourent.

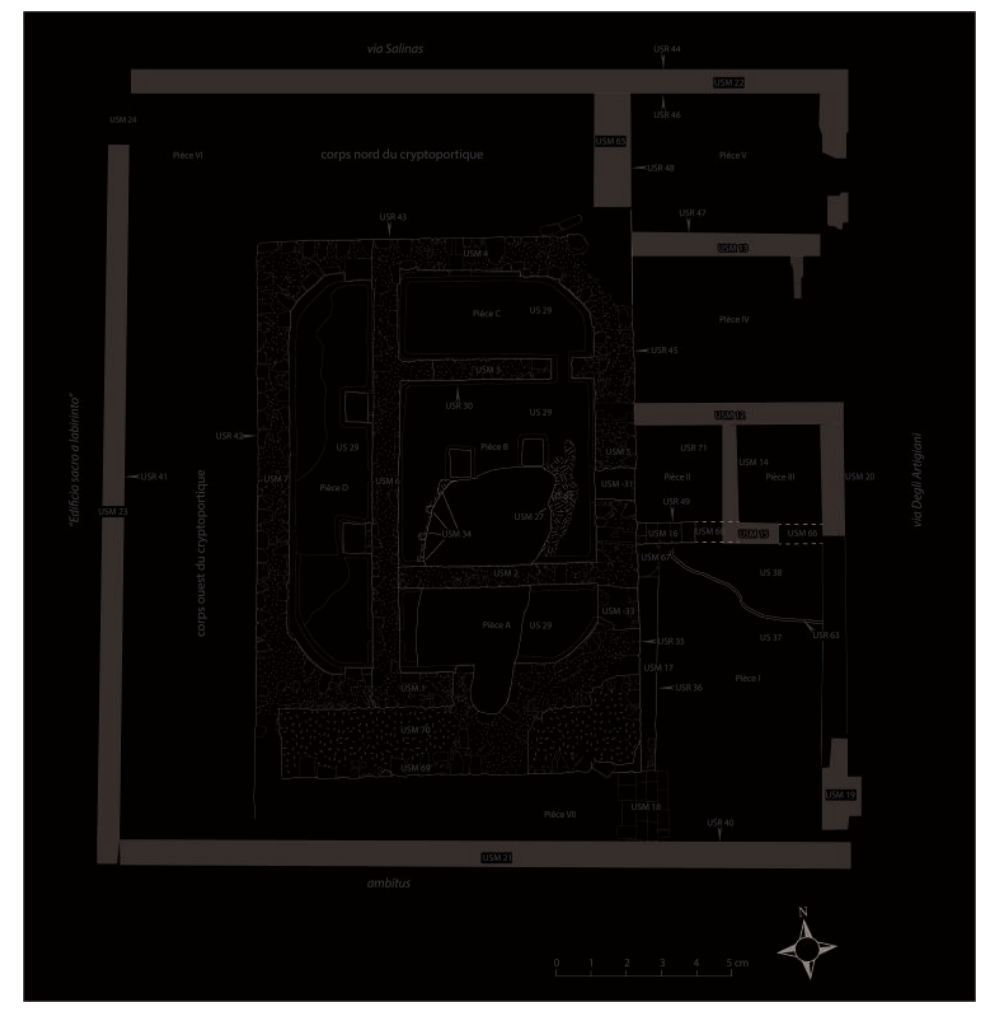

I. FUMADò ORTEgA, G. POLIZZI.

Nous avons réalisé un nouveau plan de la citerne maçonnée qui nous a permis d'observer un certain nombre de détails structurels absents des dessins de ce monument publiés jusqu'à présent ${ }^{22}$. Pourtant, ces détails sont très utiles pour la compréhension des différentes phases d'utilisation de cette citerne. Les observations que nous avons réalisées portent sur le système de couverture de la citerne maçonnée et suggèrent un changement de sa fonction, ainsi que l'existence d'un édifice antérieur à la construction de ce grand réservoir. 

par exemple les marques dans l'enduit (fig. 14) du mur nord de la pièce $C$, nous indiquent la présence d'éléments architecturaux, actuellement non conservés, destinés à soutenir la toiture de la citerne maçonnée. Une ligne entre ces marques et les piliers conservés au milieu de la pièce B est parfaitement parallèle aux murs USM ${ }^{25} 5,6$ et 7 et perpendiculaire aux murs USM 1, 2, 3 et 4 (fig. 13).

Fig. 14 - Côté sud du parement du mur USM 4 avec les traces, dans l'enduit, qui révèlent la présence d'éléments architecturaux appartenant au système de toiture.

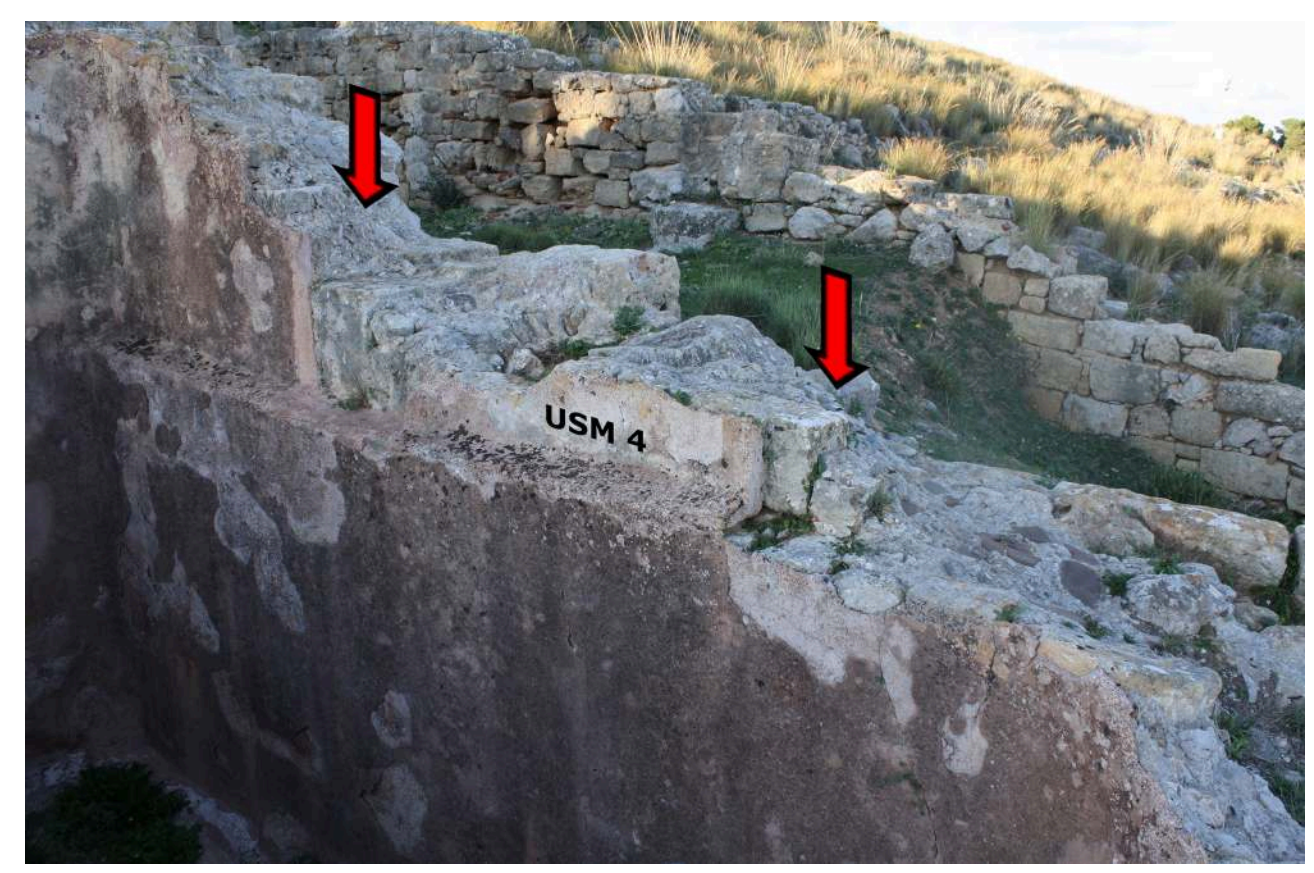

Photo G. Polizzi.

Nous pouvons compléter ainsi la reconstruction du système de couvertures de la citerne maçonnée en proposant la présence de 14 points d'appui pour cette toiture. Il est probable que ces points d'appui soutenaient des architraves, cependant, nous ne disposons pas encore de données suffisantes pour éclairer cet aspect (fig. 15). 
Fig. 15 - Restitution 3d de la citerne maçonnée. En rouge, proposition d'organisation des éléments de soutènement de la couverture.

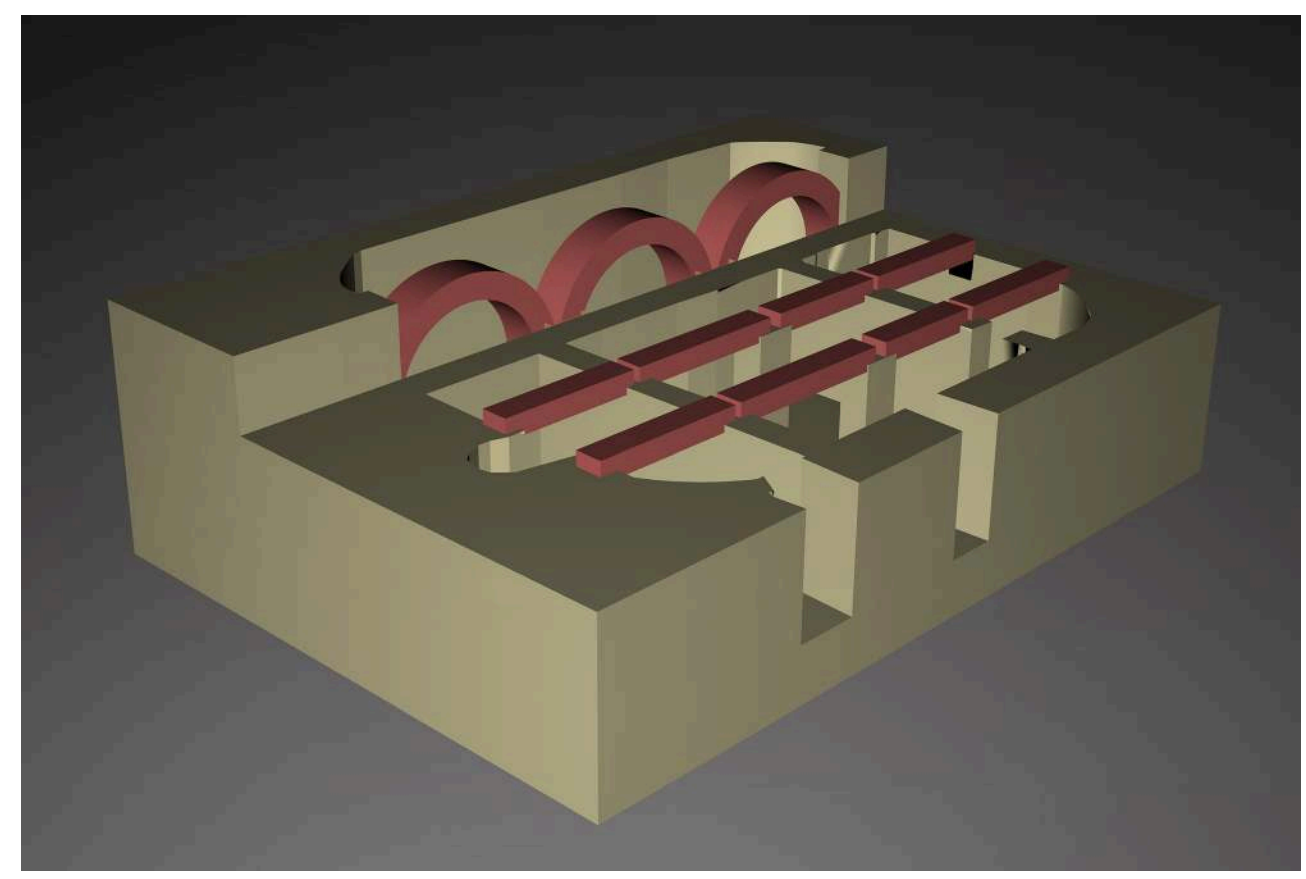

G. Polizzi.

D'autres détails indiquent l'existence de plusieurs phases de construction à l'intérieur de l'îlot XII. Le plus évident de ces indices est le remploi de matériaux de construction de divers types dans la réalisation de la citerne maçonnée. De même, la modification de certaines de ses caractéristiques structurelles, qui sera expliquée ci-dessous, est incompatible avec la fonction de réservoir d'eau. Les modifications internes à la structure indiquent une fonction variable du bâtiment au cours de sa fréquentation.

La réutilisation de certains matériaux est évidente à première vue. Les murs USM 4, 5 et 7 ont des restes de colonnes à plusieurs formats et des blocs de calcaire taillés remployés dans la maçonnerie à côté de pierres brutes de calcaire, de calcarénite et de biocalcarénite de petite et moyenne taille qui ont été légèrement démaigries du côté du parement du mur. Le remploi de ces éléments d'architecture de prestige (fig. $16 \mathrm{a}-\mathrm{b}$ ) indique la présence d'un bâtiment important dans les environs de l'îlot XII qui aurait été démantelé lors de la construction de la citerne maçonnée. 
Fig. 16 - Colonne (a) et bloc (b) remployés dans la maçonnerie du mur USM 5.
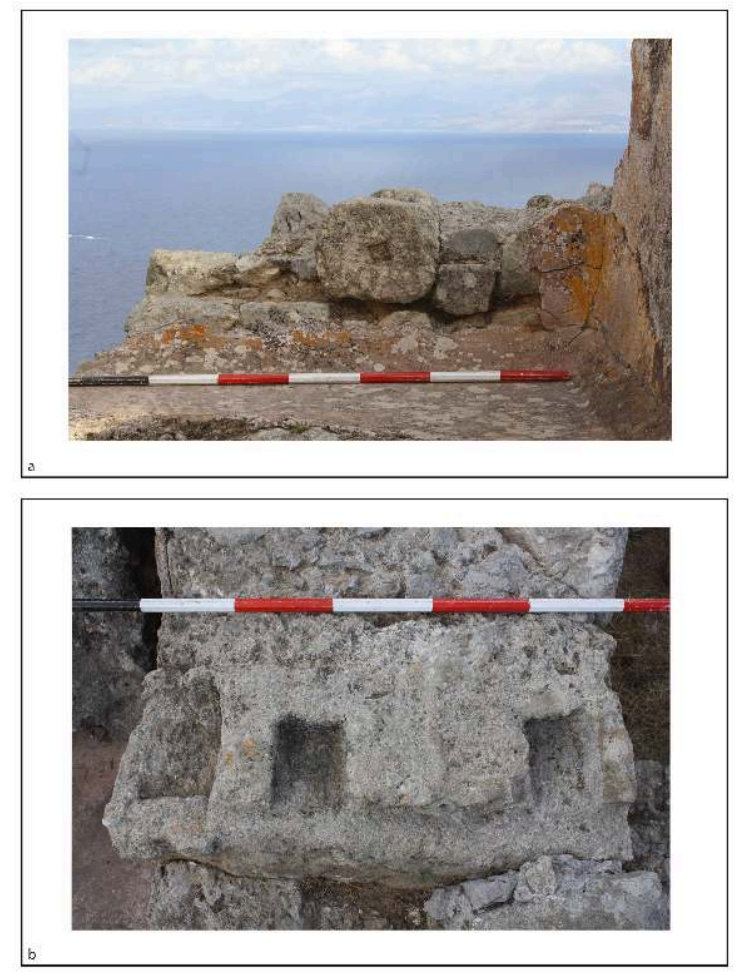

Photo G. Polizzi.

En outre, l'angle nord-ouest formé par les murs USM 4 et 7 de ladite citerne présente des blocs superposés et chanfreinés de calcarénite (fig. 17). 
Fig. 17 - Angle extérieur nord-est de la citerne maçonnée.

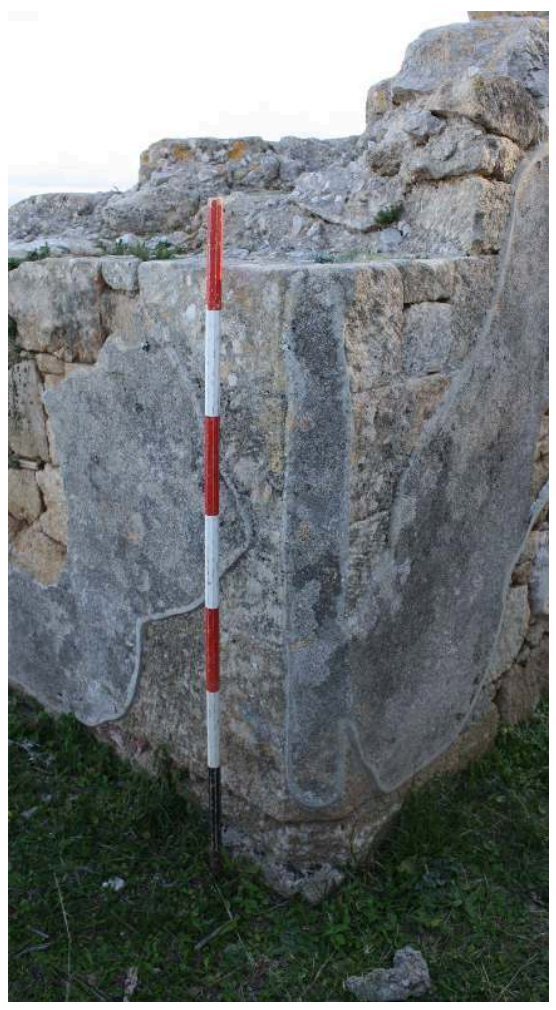

Photo G. Polizzi.

D'autres blocs chanfreinés de même origine se trouvent à l'intérieur du mur du périmètre nord de l'îlot XII et dans le corps occidental du cryptoportique qui entoure la citerne maçonnée (fig. 18). 
Fig. 18 - Pilastre chanfreiné au cryptoportique.

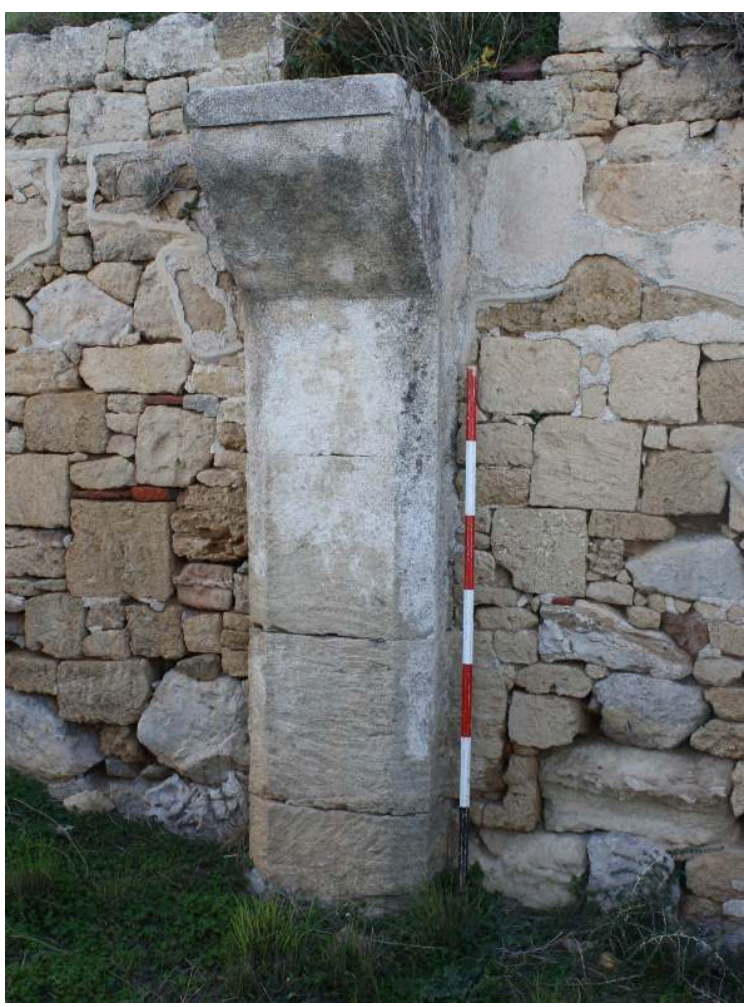

Photo G. Polizzi.

Aucun de ces blocs n'est aligné avec l'angle de la citerne dont nous parlons, c'est-à-dire qu'ils ont été tous, ou au moins une partie d'entre eux, déplacés de leur position originale. Avec les données dont nous disposons, il n'est pas possible de déterminer lesquels de ces blocs sont en position primaire et lesquels ont été remployés. Mais la disposition générale des vestiges visibles sur le site nous amène à penser que le pilastre intégré dans le mur occidental du cryptoportique (USM 78) pourrait être le seul témoin architectural qui reste in situ après le réaménagement de l'espace opéré dans l'îlot XII lors $\mathrm{du}$ démantèlement $\mathrm{du}$ premier édifice et de la construction de la citerne maçonnée ${ }^{26}$. Un parallèle sur lequel nous reviendrons nous indique le milieu du $\mathrm{II}^{\mathrm{e}}$ siècle av. J.-C. comme date approximative pour la construction de l'édifice qui a précédé la citerne maçonnée.

Des épisodes de remploi sont observables encore sur le mur USM 2 qui traverse la cavité de la roche (fig. 13). Outre des blocs de calcarénite, on trouve des morceaux remployés d'un béton de tuileau avec couche de préparation ayant des caractéristiques très proches de ceux de l'USR 30 et USR 72. Cela pose des questions sur la contemporanéité des murs USM 1, 4, 5 et 6, des murs USM 2, 3 et 7, qui présentent des caractéristiques très similaires mais qui se juxtaposent aux autres.

En outre, il y a deux types d'arguments qui témoignent de la fin de l'utilisation de la structure comme réservoir d'eau et qui n'avaient pas été mentionnés dans les publications antérieures. Il s'agit, d'une part, de l'ouverture de deux baies (US 31 et US 33), très vraisemblablement des portes, dans la partie inférieure de la paroi de la citerne maçonnée (USM 5) qui donne sur les pièces I, II et IV qui, à leur tour, s'ouvrent sur la Via degli Artigiani. D'autre part, on observe des indices de l'installation d'une couverture pour la cavité rocheuse (US 27), probablement en bois, au niveau du 
pavement de la pièce $B$ de la citerne maçonnée. Nous allons commenter rapidement ces deux types de vestiges.

L'ouverture de deux portes dès l'époque antique dans la citerne maçonnée est confirmée par deux éléments : le premier est le fait que le solin d'étanchéité présent dans toutes les jonctions entre les parois et le sol de la citerne maçonnée a été soigneusement enlevé en correspondance des deux ouvertures pratiquées sur l'USM 5 (fig. 19). Les surfaces inférieures de ces baies ont été arasées jusqu'à des cotes légèrement inférieures à celles des pavements intérieurs des pièces $\mathrm{A}$ et $\mathrm{B}$, sur lesquelles elles ouvrent ${ }^{27}$.

Fig. 19 - Solin d'étanchéité arasé en face de la porte US 31 ouverte dans le mur USM 5.

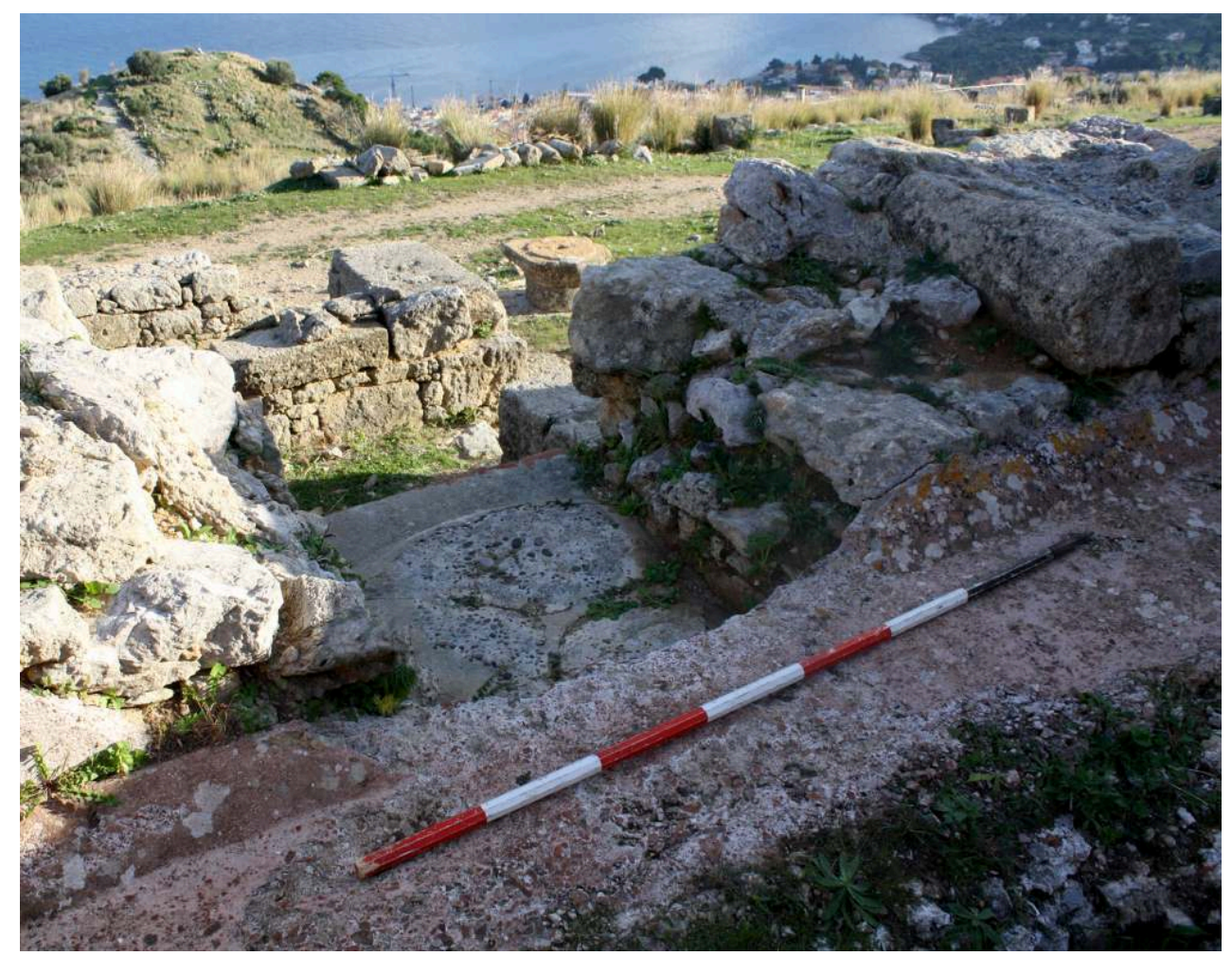

Photo G. Polizzi.

Ensuite, la surface de la baie US 31 a été couverte par un pavement en béton de galets (fig. 20) qui, après une inspection autoptique préliminaire, montre les mêmes caractéristiques que celui appliqué dans la pièce I (fig. 21) ${ }^{28}$. 
Fig. 20 - Seuil US 31 en béton de galets aménagé sur le mur USM 5.

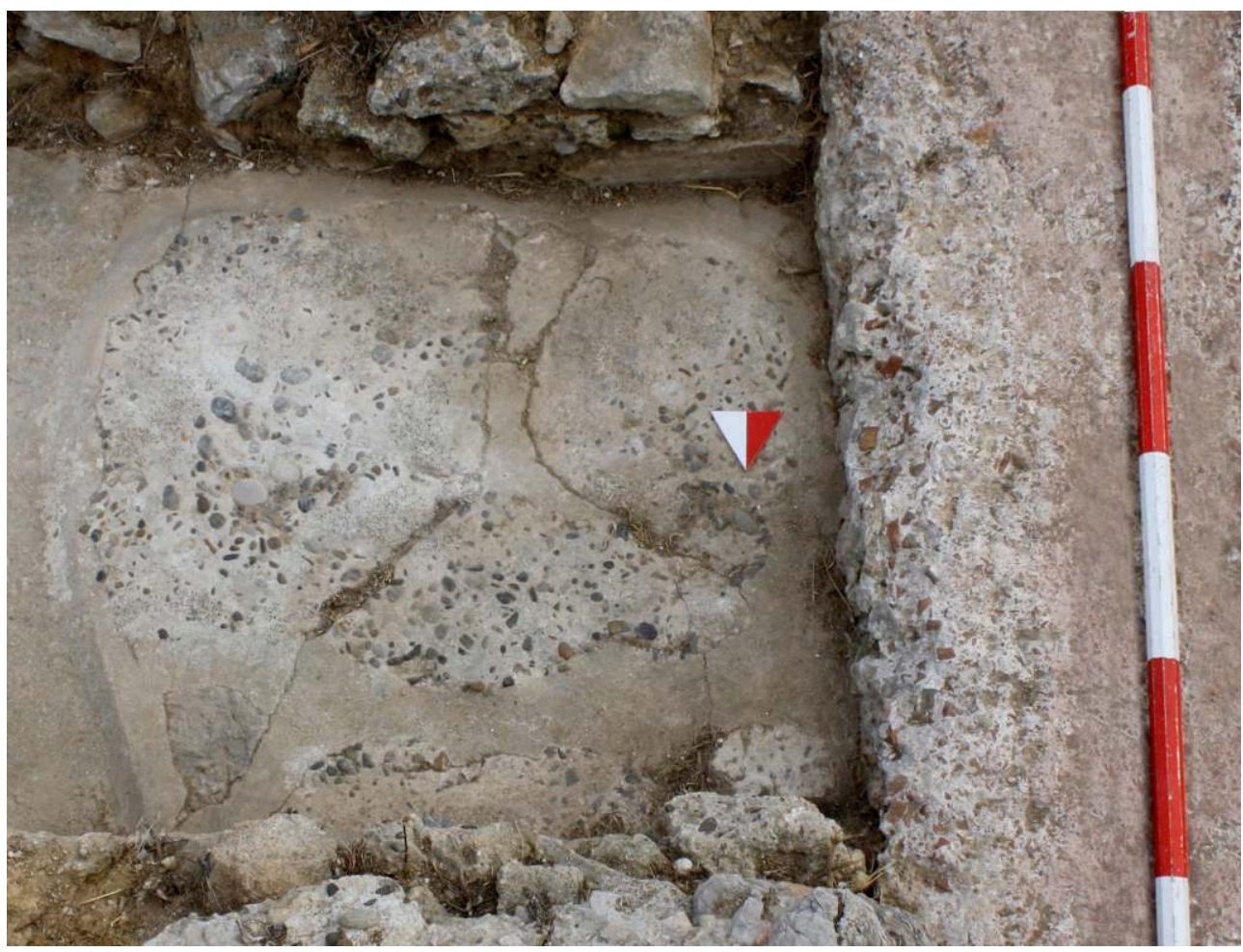

Photo G. Polizzi.

Fig. 21 - Pièce I. Sol en béton de galets.

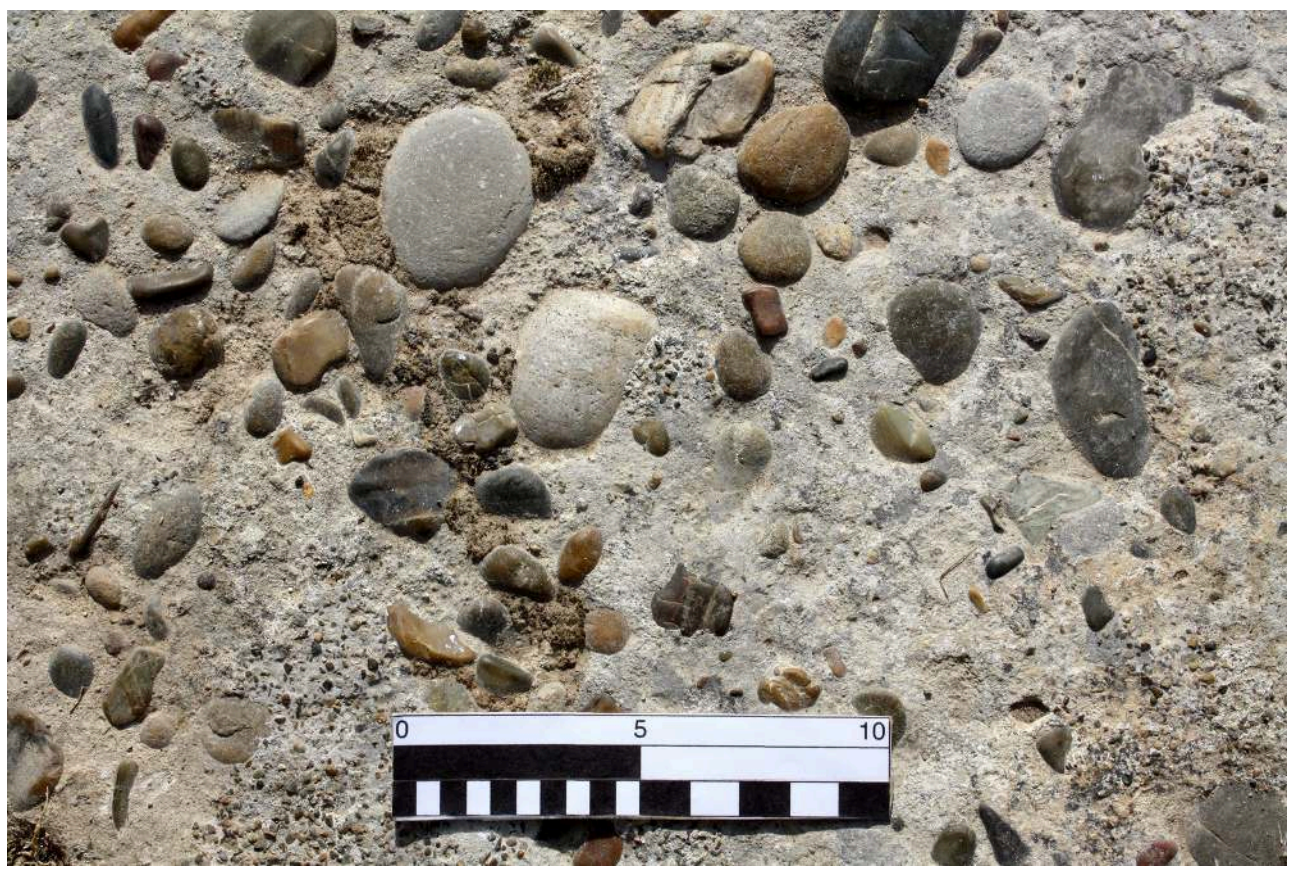

Photo G. Polizzi.

Le deuxième élément qui confirme que ces baies sont des portes, est l'installation d'une couverture plane en bois sur la cavité rocheuse (US 27). À l'intérieur de la pièce B, sur l'extrémité ouest de cette cavité, une série de coupures (US 34) d'environ 15 x 18 x 17 
$\mathrm{cm}$ ont été pratiquées sur le pavement de la pièce (fig. 13). Si sur le dernier plan publié on n'apprécie que trois de ces coupures ${ }^{29}$ en disposition presque radiale, après le nettoyage d'août 2016 une quatrième coupure, orthogonale et plus grande (40 x 27 x 19 $\mathrm{cm})$, a été mise en évidence (fig. 22).

Fig. 22 - Coupures faites dans le pavement de la pièce B de la citerne maçonnée.

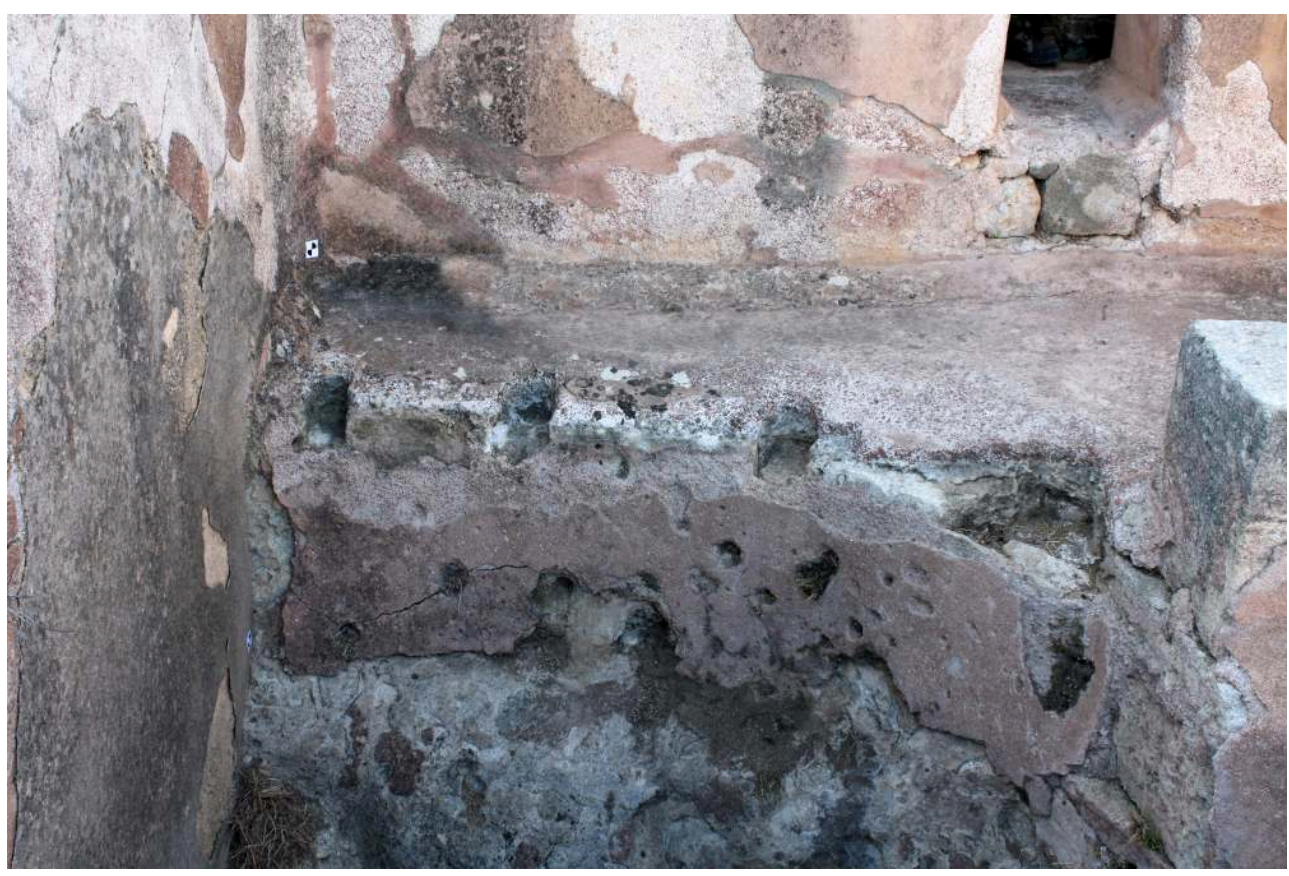

Photo G. Polizzi.

Ces quatre niches semblent répondre à la nécessité d'installer des poutres afin de couvrir la cavité rocheuse, de plus de $3 \mathrm{~m}$ de profondeur, permettant ainsi la circulation de personnes et/ou d'animaux à l'intérieur de la pièce $B$ sans risquer d'y tomber. Ces opérations sont évidemment incompatibles avec la fonction de stockage d'eau à l'intérieur des pièces de la citerne maçonnée. Pourtant, rien n'empêche que la cavité rocheuse (US 27) ait pu continuer à être utilisée comme citerne après le réaménagement final du bâtiment.

30 L'une des caractéristiques de la citerne maçonnée est le bon état de conservation de ses revêtements pariétaux, dont l'analyse archéométrique et archéologique va nous permettre de comprendre l'histoire de sa construction.

31 Une inspection préliminaire des enduits nous indique que les multiples différences de couleurs et de nuances proviennent, dans de nombreux cas, de l'effet des conditions d'exposition à la lumière du soleil et à l'humidité, en fonction de l'orientation des murs (fig. 23a). Parfois, celles-ci relèvent de la variété des matériaux utilisés. Une première analyse macroscopique des parois intérieures de la citerne maçonnée nous a permis de distinguer un total de huit types d'enduits ${ }^{30}$. Ceux-ci présentent une différence qui relève de l'utilisation du sable de plage ou de rivière pour la réalisation des mélanges. Cinq enduits sont faits avec du sable de plage ${ }^{31}$ (fig. 23b). Chacun d'entre eux a des caractéristiques très différentes en matière de traitement final de la surface ${ }^{32}$, de granulométrie des agrégats ${ }^{33}$ et de résistance du mélange ${ }^{34}$. 
Fig. 23 - Orthophotographie du parement est (a) et relevé des enduits présents sur le parement est du mur USM 6 dans la pièce $B(b)$.
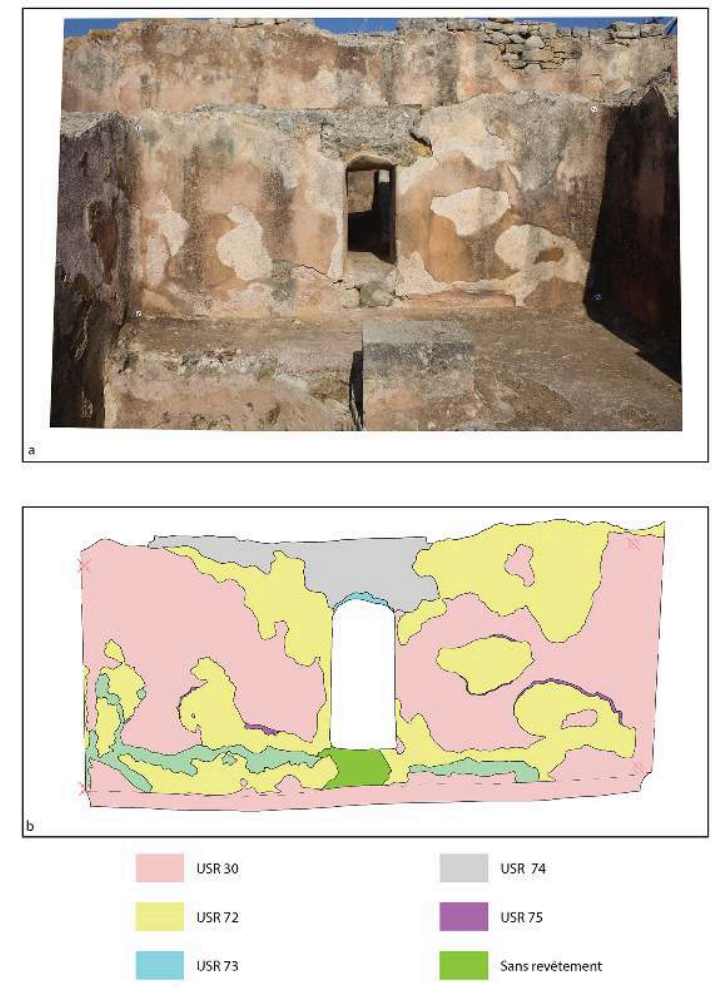

\section{Fumadò Ortega.}

Ces revêtements ont été également appliqués dans des points particuliers, principalement dans les zones de fracture des enduits faits à base de sable de rivière. L'observation de leur stratigraphie verticale confirme que ces cinq enduits sont les derniers à avoir été appliqués sur les parois de la citerne maçonnée. Par ces raisons, nous croyons que ces cinq revêtements ne sont pas antiques mais sont le résultat de diverses opérations de restauration. Si l'enduit USR 77 et sa couche de préparation (USR 76) peuvent correspondre aux techniques de restauration utilisées dans les années 1960 et 1970, l'USR 73, plus élaborée et avec un graphite italien en calligraphie " ancienne ", pourrait être le résultat d'une première opération de restauration datant de la première moitié du XX ${ }^{\mathrm{e}}$ siècle ou de la fin du XIX $\mathrm{X}^{\mathrm{e}}$ siècle.

Trois autres enduits sont faits de sable de rivière ${ }^{35}$. L'un d'entre eux, l'USR 72, est une couche de préparation. Celle-ci est un mortier de 3 à $4 \mathrm{~cm}$ d'épaisseur contenant du sable grossier et des fragments de céramique de taille hétérogène, de 2 à $10 \mathrm{~mm}$ de diamètre, mélangés avec du mortier très blanc et très compact. Au-dessus de cette préparation une couche de mortier de tuileau (USR 30) a été étalée. (fig. 24a-b). Dans l'état actuel de conservation de la citerne maçonnée, cette couche couvre la plupart de l'intérieur des parois du réservoir. Il s'agit d'un mélange rosâtre, très homogène et résistant, de 0,5 à $1 \mathrm{~cm}$ d'épaisseur, composé principalement de mortier, de sable de rivière et de fragments de céramique plus fins, de 2 à $4 \mathrm{~mm}$ de diamètre. 
Fig. 24 - Revêtements USR 72 couvert par le mortier de tuileau USR 30 (a) et revêtements USR 72 couvert par des enduits de restauration (b).
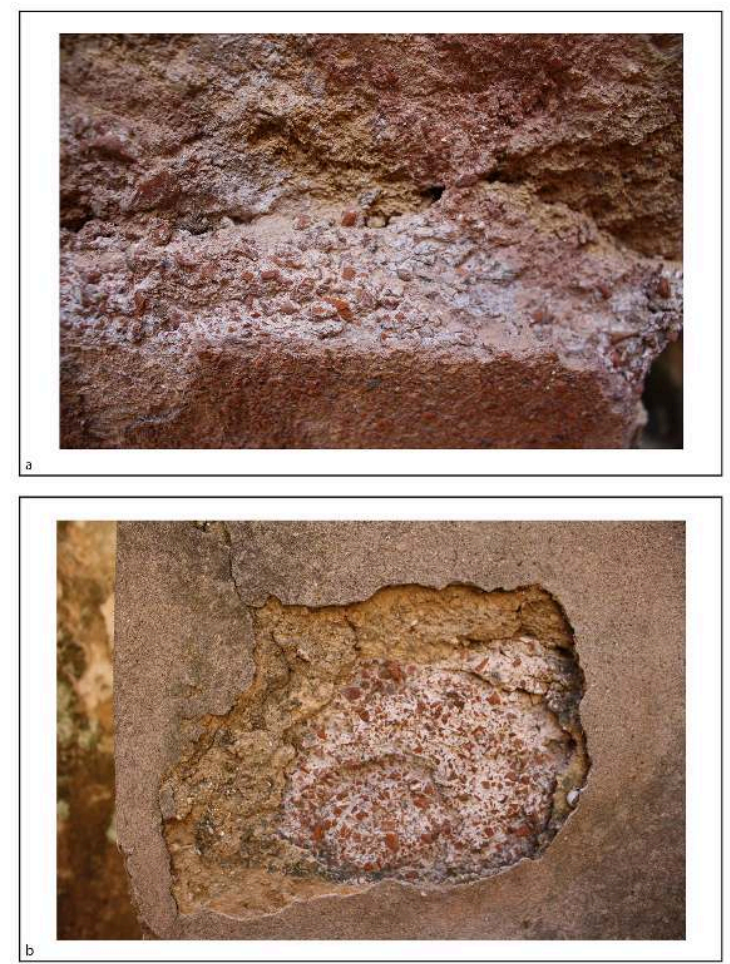

Photo G. Polizzi.

Le dernier enduit, l'USR 28, se distingue clairement du mortier de tuileau antérieur par sa couleur, par sa composition et par les points où il a été appliqué. L'USR 28 a une couleur jaune clair, ne revèle aucun fragment de céramique et n'a pas été appliqué sur les murs de la citerne maçonnée, mais seulement sur les parois de la cavité rocheuse (US 27). Il faut souligner la forte concentration de sable de rivière et l'homogénéité de son grain. Stratigraphiquement, l'USR 28 est le plus ancien des huit enduits mentionnés et trouve des parallèles très proches dans la plupart des réservoirs d'eau du Monte Catalfano ${ }^{36}$.

À la lumière de ces observations, nous avons considéré que seuls les trois enduits contenant $\mathrm{du}$ sable de rivière étaient antiques. En attendant des données archéométriques et de nouvelles analyses provenant des futures campagnes de terrain, nous proposons l'hypothèse que ces trois revêtements correspondent aux deux horizons chronologiques suivants :

1. Dans le premier, seul l'enduit USR 28 a été utilisé et seulement dans la cavité rocheuse (US 27), alors aménagée comme réservoir d'eau. On peut supposer l'existence d'un bâtiment sur cette citerne. Nombre de maisons sur le Monte Catalfano, comme celles du quartier périphérique ${ }^{37}$, celle de la "Casa del corridoio $»^{38}$ ou celles qui sont situées dans l'îlot de l'édifice connu comme "aire sacrée à trois bétyles " ${ }^{39}$, parmi d'autres exemples, montrent une situation similaire, dans laquelle des failles dans la roche avaient été aménagées et revêtues d'un mortier aux caractéristiques très similaires à celles de l'USR 28, pour accueillir des réservoirs d'eau privés à forme irrégulière.

2. Dans le deuxième des horizons chronologiques proposés, la citerne maçonnée a été 
construite en englobant la citerne préexistante dans la cavité rocheuse. Tout l'intérieur de la nouvelle structure a été alors recouvert avec du mortier de tuileau (USR 30).

\section{Périodisation}

À partir des données actuellement disponibles, nous proposons une hypothèse préliminaire sur les phases de construction de l'espace occupé par la citerne maçonnée.

\section{Première phase :}

Nous considérons comme probable que l'architecture des blocs de calcarénite chanfreinés ait appartenu à un bâtiment d'un certain prestige construit sur une cavité de la roche. Ce bâtiment, actuellement disparu, était doté d'un réservoir d'eau de $30 \mathrm{~m}^{3}$ logé dans cette même cavité. À cette phase correspondrait l'enduit le plus ancien, notre USR 28.

\section{Deuxième phase :}

37 À un certain moment ce bâtiment aurait été démantelé et remplacé par la citerne maçonnée, celle-ci réutilisant des éléments constructifs de l'ancien édifice. Cette nouvelle et très grande citerne a permis de multiplier par sept la capacité de stockage d'eau du réservoir précédent, en l'englobant dans sa structure et en le dotant d'une capacité di circa $230 \mathrm{~m}^{3}$. La citerne maçonnée a été complètement imperméabilisée par une couche de mortier de tuileau, notre USR 30. Cette couche a été appliquée dans la cavité de la roche directement sur l'enduit antérieur, préalablement piqueté, et sur une couche de préparation (USR 72) dans les parois du nouveau bâtiment.

\section{Troisième phase :}

Lors d'une phase postérieure la citerne maçonnée perd sa fonction de réservoir d'eau. Son intérieur est rendu accessible et praticable avec deux connexions vers l'extérieur au niveau de son pavement. On ne peut pas se prononcer sur la continuité du stockage d'eau dans la cavité de la roche (US 27) pendant cette phase, mais c'est une hypothèse plausible. Sans connaître les détails des fouilles anciennes, nous ignorons quelles ont été les circonstances et le moment précis de l'abandon définitif de ce bâtiment ${ }^{40}$.

\section{Chronologie}

En attendant de nouvelles données archéométriques et de nouvelles fouilles, les seuls indices chronologiques de cette séquence relative proviennent de l'étude architecturale menée par Markus Wolf sur l'agora. Les jambages de l'escalier nord de la stoa, encore en place, présentent un chanfrein très similaire à celui des blocs remployés dans la citerne maçonnée (fig. 25). 
Fig. 25 - Blocs du jambage de l'escalier nord de la stoa.

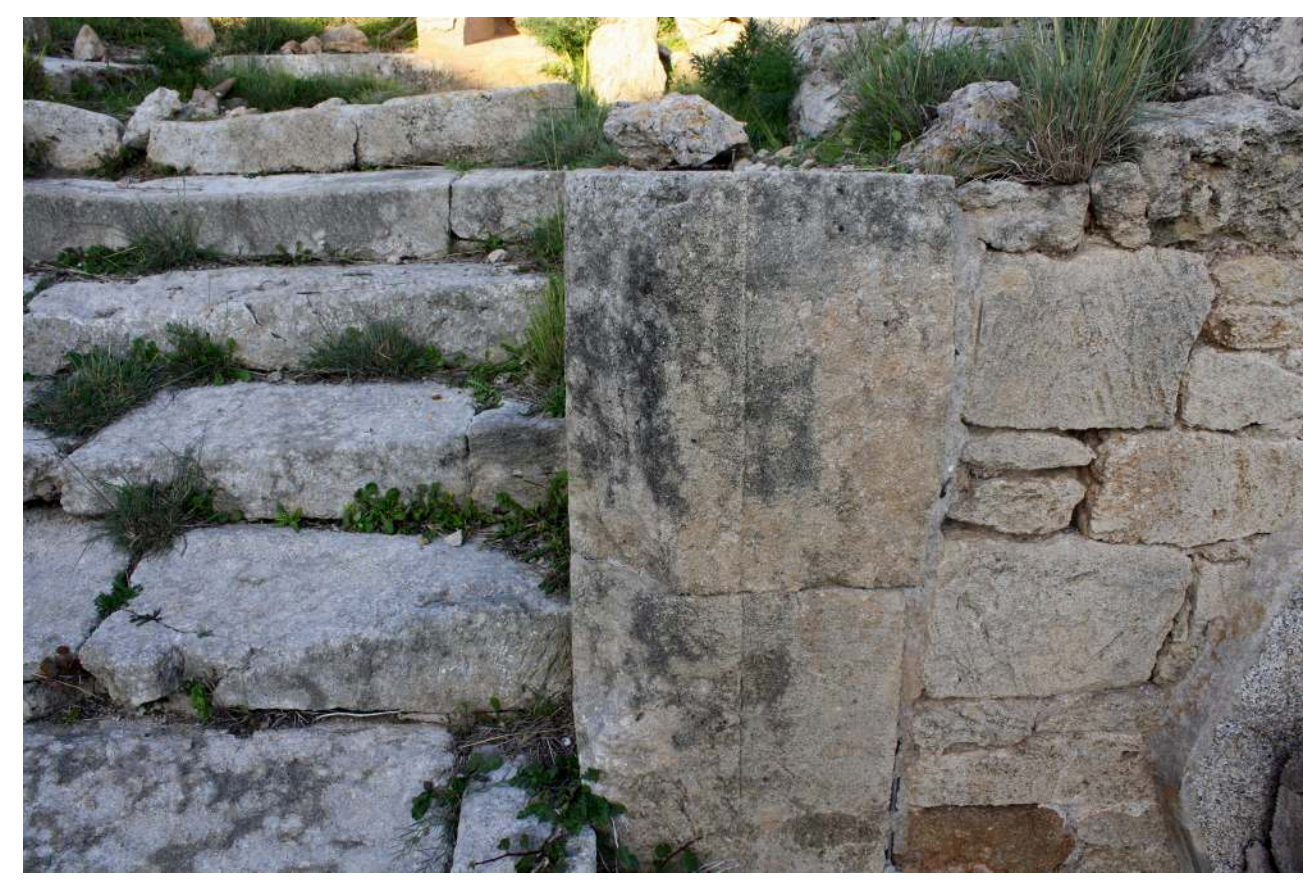

Photo G. Polizzi.

La construction de la stoa a été datée au milieu du II ${ }^{\mathrm{e}}$ siècle av. J.-C. ${ }^{41}$ Nous adoptons donc, de façon préliminaire, cette fourchette chronologique pour notre première phase, antérieure à la construction de la citerne maçonnée. Celle-ci, ainsi que son changement de fonction, doit être placée entre la deuxième moitié du II ${ }^{\mathrm{e}}$ siècle av. J.-C. et l'abandon définitif du monte Catalfano. De prochaines campagnes de terrain et l'étude des documents produits lors des fouilles anciennes pourront nous aider à vérifier ces hypothèses.

\section{Discussion et conclusion}

L'ensemble de ces nouvelles données nous permet d'avancer quelques conclusions préliminaires sur le lien, en apparence étroit, entre le contexte hydrogéologique du Monte Catalfano et la gestion de l'eau dans la cité de Solonte. La lithologie et les nombreuses fractures ouvertes des affleurements carbonatés et dolomitiques conséquentes à la tectonique Plio-Quaternaire, constituent une des principales modalités de recharge de l'aquifère (infiltration des eaux météoriques et pertes éventuelles des cours d'eau locaux). Ce dernier dispose d'importantes capacités de stockage et de recharge. Le contexte morphostructural et tectonique caractéristique de ce secteur, où dominent une forte porosité et des tensions à l'origine d'une intense fracturation, a eu pour conséquence hydrogéologique le développement d'émergences au potentiel hydrothermal. Les modes d'exsurgence et de concrétionnement peuvent, dans certains cas, s'apparenter à ceux rencontrés dans le cas de travertins thermogènes d'autres régions d'Italie ${ }^{42}$. Une des spécificités du site de Solonte proviendrait également d'un système aquifère localement compartimenté par des failles plus ou moins profondes. Celles-ci pourraient orienter les axes de drainage de la nappe et favoriser la concentration des remontées de type hydrothermal dans un espace régional circonscrit au Monte Catalfano. Un mélange d'eaux de recharge et 
hydrothermales profondes avant l'émergence alimenterait in fine certaines citernes et bassins et de manière plus importante encore, les petits thermes. Un des facteurs ayant influencé le choix de l'implantation de la cité de même que l'organisation de son système de gestion des eaux, pourrait alors être d'ordre hydrogéologique.

Ces premières constatations nécessitent un effort de recherche plus approfondie et soulèvent de nombreuses questions. Dans un contexte climatique de type méditerranéen à longue saison sèche et modeste pluviométrie (souvent concentrée sur de courtes périodes ou des épisodes extrêmes), et sur un site aux affleurements calcaires faillés et karstifiés, un apport pérenne en eau paraît nécessaire. Notamment pour le développement et la prospérité d'une cité de l'envergure de Solonte (dotée d'une population de plusieurs milliers d'habitants et de nombreuses infrastructures dédiées à l'utilisation de l'eau comme les thermes et les bassins, dont un ${ }^{43}$ présente une capacité de $800 \mathrm{~m}^{3}$ ). La seule récolte des eaux météoriques pourrait paraître insuffisante. Il convient toutefois de nuancer ce bilan car on ne trouve pas systématiquement de traces des micro-exsurgences à l'intérieur des citernes, recouvertes de mortier hydraulique. Cependant, en de nombreux endroits du site, des concrétionnements se sont développés au niveau des conduites et cannelures de même que sur certaines surfaces rocheuses. Ceci indique clairement que les eaux de ruissellement, de débord ou de trop plein de nombreux dispositifs de collecte, sont d'origine profonde. D'ailleurs dans les localités toutes proches de Villabate et de Pizzo Cannita, des remontées hydrothermales sont connues $^{44}$. Les nappes y trouvent leur niveau phréatique entre $33 \mathrm{~m}$ et $15 \mathrm{~m}$ de profondeur ${ }^{45}$. Les eaux sont fortement carbonatées et ont des températures estimées autour de $20 / 30^{\circ} \mathrm{C}^{46}$. Cette constatation permet d'envisager qu'à Solonte certaines citernes/bassins, et notamment les thermes, aient été alimentés par des eaux thermominérales $\left(>\right.$ de $5^{\circ} \mathrm{C}$ à la température moyenne de la zone d'émergence) ${ }^{47}$.

Au niveau de l'évolution des conditions environnementales, des exsurgences ou griffons ont pu se tarir ou voir leur parcours se modifier lors d'épisodes de forte sismicité, fréquents dans la région ${ }^{48}$. Ce qui aurait engendré la nécessité de modifier certaines structures et/ou de trouver des compléments au niveau des collecteurs. L'occurrence de ces évènements de même que leur influence sur la phase d'abandon final de la cité est une question qui mérite d'être traitée. Ceci doit également être considéré au niveau de l'évolution des régimes de précipitations sur la longue durée (variation dans leur répartition ou récurrence d'épisodes de sécheresse) bien que, compte tenu de la nature profonde des réservoirs et de l'étendue de leur alimentation (drainage des massifs et collecte en direction du niveau de base marin), il existe une certaine inertie par rapport à la variable climatique/météorologique. Certains modèles expérimentés dans le sud de l'Italie sur calcaires dolomitiques indiquent un temps de pénétration des eaux de pluies (en volume et non en masse) avant d'atteindre la nappe, de 0 à 210 jours $^{49}$. Ce fonctionnement en système inertiel est également à considérer au niveau des sources profondes lors de la restitution. Une bonne continuité dans l'alimentation en eau de la cité, sauf cas extrême, parait donc assurée. La faiblesse des remontées actuelles à Solonte, si l'on ne considère pas un éventuel impact imputable aux séismes anciens, serait à rechercher au niveau de la surexploitation des eaux de surface, de la zone vadose, et des aquifères peu profonds venant alimenter les réservoirs hydrothermaux. 


\section{Solonte à ce sujet se retrouvent dans d'autres sites contemporains de Sicile. Pour exemple, à Monte Adranone (Sambuca di Sicilia), Monte Iato (San Cipirello) et Morgantina (Aidone), les contextes bioclimatiques et morphostructuraux sont proches de ceux rencontrés à Solonte. Ces sites sont positionnés sur des reliefs; ils ne disposent pas de sources naturelles évidentes et les citernes et bassins aux capacités de stockage les plus importantes sont localisés à proximité ou au niveau des espaces publics ${ }^{50}$. La question de l'éventualité d'une alimentation additionnelle en eau, selon des caractéristiques proches ou identiques à celles de Solonte pourrait alors se poser. \\ Enfin, l'étude approfondie des citernes a également permis d'identifier de possibles modifications dans l'implantation urbaine. À titre d'exemple, dans la partie occidentale de la ville, certaines citernes ( $n^{\circ} 58,59$ et 37 ) ont été séparées en deux au cours de divisions de parcelles. Ainsi, parallèlement à nos recherches, d'importants témoignages concernant l'organisation urbaine et la propriété des eaux sont mis en évidence et peuvent être exploités pour une meilleure connaissance des politiques de gestion de l'eau dans le contexte antique de la cité de Solonte.}

convient également de noter que plusieurs des paramètres et des situations définies à

\section{BIBLIOGRAPHIE}

Agenza Regionale per i Rifiuti e le Acque, Carta delle isoiete per il periodo 1921-2003, http://

www.osservatorioacque.it/dati/cartepluviotermo/isoiete_1921-2003.pdf.

Amico 1757 = V.M. Amico, Lexicon topographicum siculum, Palerme,1757, éd. italienne 1855-1856, trad. et note de G. Di Marzo.

Armenio et alii 2015 = D. Armenio, G. Di Rosa, A. Lo Bello, V. Cutrone, L. Geraci, G. La Placa, A. Licciardello, A. Margagliotta, M. Territo, Piano di Gestione 2015-2021, Distretto idrografico della Sicilia, Documento preliminare, 2015.

Catalano et alii 2013 = R. Catalano, G. Avellone, L. Basilone, A. Contino, M. Agate, Carta geologica d'Italia, Palerme, foglio 595, 1 :25.000, Palerme, 2013.

Bell 2012 = M. Bell III, Spazio e istituzioni nell'agorà greca di Morgantina, dans C. Ampolo (dir.), Agorà greca e agorai di Sicilia, Pise, 2012, p. 111-118.

Berardi et alii 2016 = G. Berardi, G. Vignaroli, A. Billi, F. Rossetti, M. Soligo, S. Kele, M. O. Baykara, S. M. Bernasconi, F. Castorina, F. Tecce, C. Shen, Growth of a Pleistocene giant carbonate vein and nearby thermogene travertine deposits at Semproniano, southern Tuscany, Italy: estimate of $\mathrm{CO}^{2}$ leakage, dans Tectonophysics, 30, 2016, p. 219-239.

Bottari - Stiros - Teramo 2009 = C. Bottari, S. C. Stiros, A. Teramo, Archaeological evidence for destructive Earthquakes in Sicily between 400 B.C. and A.D. 600, dans Geoarchaeology, 24, 2009, p. $147-175$.

Cavallari 1875 = F. S. Cavallari, Posizione topografica di Solunto, dans Bullettino della Commissione di antichità e belle arti in Sicilia, VIII, 1875, p. 1-10.

Chronique des activités archéologiques de l'École française de Rome, Sicile 
Climate-Data.org, Diagramme ombrothermique de Bagheria, https://fr.climate-data.org/location/ 13874/.

Cutroni Tusa et alii 1994 = A. Cutroni Tusa, S. Tusa, A. Italia, D. Lima, Solunto, Rome, 1994.

Fiorillo - Pagnozzi 2015 = F. Fiorillo, M. Pagnozzi, Recharge processes of Matese karst massif (southern Italy), dans Environmental Earth Sciences, 2015, DOI 10.1007/s12665-015-4678-y.

Fiorillo 2011 = F. Fiorillo, Tank-reservoir emptying as a simulation of recession limb of karst spring hydrographs, dans Hydrogeology Journal, 19, 2011, p. 1009-1019.

Ginouvès et alii 1985 = R. Ginouvès, P. Gross, R. Martin, F. Coarelli, C. Krause, J. Coulton, A. M. Guimier-Sorbets, Dictionnaire d'architecture, tome 1, Matériaux, techniques de construction, techniques et formes du décor, Rome, 1985.

Greco 2005 = C. Greco, Solunto : guida breve, Palerme, 2005.

Fiorentini 1980-1981 = G. Fiorentini, Ricerche archeologiche nella Sicilia centro-meridionale, dans Kokalos, XXVI-XXVII, 1980-1981, p. 581-582.

Harrison et alii 2002 = R. W. Harrison, W. L. Newell, M. Necdet, Structure and Genesis, Karstification along an active fault zone in Cyprus, dans E. L. Kuniansky (dir.), U.S., Geological Survey Karst Interest Group, Water-Resources Investigations Report 02-4174, Atlanta, 2002, p. 45-48.

Isler - Spatafora 2004 = H. P. Isler, F. Spatafora, Monte Iato : guida breve, Palerme, 2004.

Pentecost - Jones - Renaut 2003 = A. Pentecost, B. Jones, R. W. Renaut, What is a hot spring ?, dans Canadian Journal of Earth Sciences, 40, 2003, p. 1443-1446.

Polizzi sous presse = G. Polizzi, Gestione ed uso dell'acqua a Solunto : le cisterne, dans S. Bouffier, $\mathrm{O}$. Belvedere, S. Vassallo (dir.), Installations hydrauliques et gestion de l'eau en Méditerranée préromaine [HYDRSMED Symposium II, Università di Palermo, décembre 2015], Aix-en-Provence, sous presse.

Portale 2007 = E. C. Portale, Per una rilettura del II stile a Solunto, dans F. Zevi, J.-M. Moret, A. Pelletier (dir.), Villas, maisons, sanctuaires et tombeaux tardo-républicains : découvertes et relectures récentes [Actes du colloque international de Saint-Romain-en-Gal en l'honneur d'Anna Gallina Zevi (Vienne - Saint-Romain-en-Gal, 8-10 février 2007)], Rome, 2007, p. 281-311.

Salemi Pace 1872 = G. Salemi Pace, Solunto ossia le rovine di un'antica città sul Monte Catalfano, dans Nuovi Annali di costruzioni, arti ed industrie, Palerme, 1872.

Schoen 2015 = F. Schoen, "Auf dass ihnen das Wasser nicht ausgeht » : Antike Kleinwasserspeicher im zentralen Mittelmeerraum, Tübingen, 2015.

Scina 1818 = D. Scinà, La topografia di Palermo e suoi contorni, Palerme, 1818.

Sposito 2014 = A. Sposito, Solunto : paesaggio, città, architettura, Rome, 2014.

Sulli et alii 2011 = A. Sulli, F. Pepe, V. Pennino, C. Lo Iacono, M. Agate, Evidences of mud volcanoes in the Palermo and Termini Gulf (N Sicily offshore), dans Geoitalia 2011 [VII Forum italiano di scienze della Terra (Torino, 19-23 settembre 2011)] = Epitome, 3, 88.

Tusa $1972=$ V. Tusa, Solunto nel quadro della civiltà punica della Sicilia Occidentale, dans Kokalos, 1972, V, 17, p. 27-44.

Wolf $2013=$ M. Wolf, Die Agora von Solunt, Wiesbaden, 2013. 


\section{NOTES}

1. Cutroni Tusa et alii 1994, p. 12-16.

2. Une thèse est en cours sur le sujet: G. Polizzi, «L'exploitation des ressources en eau dans la cité antique de Solonte (Sicile/Italie) »(Aix-Marseille Université).

3. Catalano et alii 2013.

4. Agenza Regionale per i Rifiuti e le Acque.

5. Armenio et alii, 2015.

6. Sulli et alii 2011.

7. Amico 1757.

8. Harrison et alii 2002.

9. Agenza regionale per i rifiuti e le acque.

10. Greco 2005, p. 40.

11. La citerne nous a été signalée par Rosa Torre, doctorante à l'Université de Messine qui étudie actuellement les thermes de Solonte.

12. Le décor pariétal présent dans la pièce nord des thermes se partage entre une moitié inférieure en cocciopesto et une partie supérieure en enduit blanc. Le même décor a été retrouvé dans les exèdres de la stoa, à l'ouest de l'agorà (fig. 12. b) et dans la pièce précédant le laconicum de la Casa del Vano Circolare (fig. 12. c). Pour la nomenclature des mortiers voir Ginouvès et alii 1985, p. 51.

13. Polizzi 2017, sous presse.

14. Cutroni Tusa et alii 1994, p. 92.

15. Sposito 2014, p. 236.

16. Cavallari 1875.

17. Voir une description préliminaire de la fouille de ce secteur dans Salemi - Pace 1872, p. 6.

18. Greco 2005, p. 4.

19. Sposito 2014, p. 230.

20. Tusa 1972, p. 32 , fig. 9.

21. Dans des campagnes de terrain suivantes nous allons analyser l'ensemble de l'insula de la maison à labyrinthe. Pour l'instant, nous nous sommes concentrés sur la citerne maçonnée.

22. Les plans publiés sont ceux de Cavallari 1875, Cutroni Tusa et alii 1994, p. 93 ; Sposito 2014, p. 237.

23. Trois d'entre eux, complets, se trouvent au fond de la citerne maçonnée. Ils mesurent 182 × 77 × $32 \mathrm{~cm}, 186 \times 81 \times 33 \mathrm{~cm}$ et $176 \times 92 \times 26 \mathrm{~cm}$.

24. Sposito 2014, 245.

25. Unità Stratigrafica Muraria

26. La vérification de cette hypothèse aurait besoin de la fouille du cryptoportique.

27. La surface de la US 33 est irrégulière et révèle une différence atteignant jusqu'à $20 \mathrm{~cm}$ de cote, selon les points de mesure. Il est probable qu'un pavement, aujourd'hui non conservé, ait été réalisé, à l'instar de l'US 31. La différence de cote entre le pavement de l'US 31 et celui de la pièce $B$ est de $6 \mathrm{~cm}$.

28. Ce pavement a été fortement restauré, ce qui nous invite à la plus grande prudence lors de son interprétation. La baie US 33 ne conserve aucun reste de pavement mais on ne peut pas exclure sa présence dans l'Antiquité.

29. Sposito 2014, p. 247.

30. USR 28, USR 30, USR 72, USR 73, USR 74, USR 75, USR 76 et USR 77.

31. USR 73, USR 74, USR 75, USR 76 et USR 77.

32. Très lissée dans l'USR 73 et rugueuse dans l'USR 77.

33. Très hétérogène dans l'USR 76 et homogène dans l'USR 77.

34. Très friable dans l'URS 75 et très compact dans l'USR 74. 
35. USR 28, USR 30 et USR 72.

36. Polizzi sous presse.

37. Cutroni Tusa et alii 1994, p. 47-49.

38. Cutroni Tusa et alii 1994, p. 51-52.

39. Cutroni Tusa et alii 1994, p. 66-70.

40. Après les fouilles des années 1868-69, 1875 et 1960-70 d'importants travaux de restauration ont été réalisés.

41. Wolf 2013, p. 41.

42. Berardi et alii 2016.

43. Polizzi sous presse.

44. Amico 1757.

45. Catalano et alii 2013, p. 183-184.

46. Scina 1818

47. Pentecost - Jones - Renaut 2003.

48. Bottari - Stiros - Teramo 2009.

49. Fiorillo 2011, Fiorillo - Pagnozzi 2015.

50. Pour les citernes publiques de Monte Adranone, voir Fiorentini 1980-81, p. 582 ; la description d'une citerne publique de Monte Jato, voir Isler, Spatafora 2004, p. 30-31; le problème du manque d'eau à Morgantina, voir Bell 2012, p. 113-114.

INDEX

Mots-clés : Sicile, Solonte, gestion de l'eau, citernes, thermes, sources, géoarchéologie Keywords : Sicily, Solunto, water management, baths, springs, geoarchaeology

\section{AUTEURS}

\section{GIOVANNI POLIZZI}

Centre Camille Jullian (Aix Marseille Univ, CNRS, Minist Culture \& Com, Aix-en-Provence, France) - giovannipolizzi[at]live.it

\section{VINCENT OLLIVIER}

LAMPEA (Aix Marseille Univ, CNRS, Minist Culture \& Com, Aix-en-Provence, France) ollivier[at]mmsh.univ-aix.fr

\section{IVÁN FUMADÓ ORTEGA}

Centre Camille Jullian (Aix Marseille Univ, CNRS, Minist Culture \& Com, Aix-en-Provence, France) - i.fumado.ortega[at]gmail.com

\section{SOPHIE BOUFFIER}

Centre Camille Jullian (Aix Marseille Univ, CNRS, Minist Culture \& Com, Aix-en-Provence, France) - sbouffier[at]mmsh.univ-aix.fr 